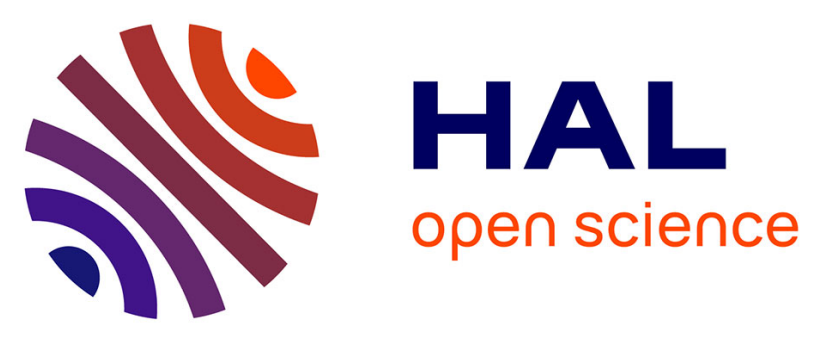

\title{
Metallic impurity content behavior during ICRH-heated L-mode discharges in EAST Metallic impurity content behavior during ICRH-heated L-mode discharges in EAST
}

\author{
G. Urbanczyk, X. Zhang, L Zhang, L. Colas, R. Dumont, W. Tierens, E. \\ Lerche, D. van Eester, Stéphane Heuraux, X. Yang, et al.
}

\section{To cite this version:}

G. Urbanczyk, X. Zhang, L Zhang, L. Colas, R. Dumont, et al.. Metallic impurity content behavior during ICRH-heated L-mode discharges in EAST Metallic impurity content behavior during ICRHheated L-mode discharges in EAST. Nuclear Fusion, 2020, 10.1088/1741-4326/abae82 . hal-02916054

\section{HAL Id: hal-02916054 \\ https://hal.univ-lorraine.fr/hal-02916054}

Submitted on 17 Aug 2020

HAL is a multi-disciplinary open access archive for the deposit and dissemination of scientific research documents, whether they are published or not. The documents may come from teaching and research institutions in France or abroad, or from public or private research centers.
L'archive ouverte pluridisciplinaire HAL, est destinée au dépôt et à la diffusion de documents scientifiques de niveau recherche, publiés ou non, émanant des établissements d'enseignement et de recherche français ou étrangers, des laboratoires publics ou privés.

\section{(ㅇ)(1) $\$$}

Distributed under a Creative Commons Attribution - NonCommercial - NoDerivatives $\mid 4.0$ 
ACCEPTED MANUSCRIPT

Metallic impurity content behavior during ICRH-heated L-mode discharges in EAST

To cite this article before publication: Guillaume Urbanczyk et al 2020 Nucl. Fusion in press https://doi.org/10.1088/1741-4326/abae82 


\title{
Metallic impurity content behavior during ICRH-heated L-mode discharges in EAST
}

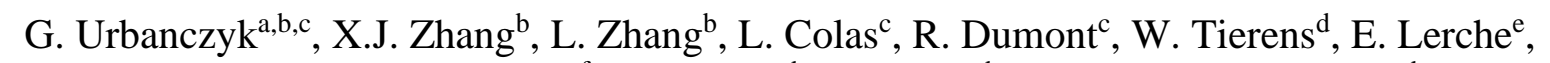
D. Van Eester ${ }^{\mathrm{e}}$, S. Heuraux ${ }^{\mathrm{f}}$, X.D. Yang ${ }^{\mathrm{b}}$, C.M. Qin ${ }^{\mathrm{b}}$, A. Ekedahl ${ }^{\mathrm{c}}$, Y.P. Zhao ${ }^{\mathrm{b}}$,

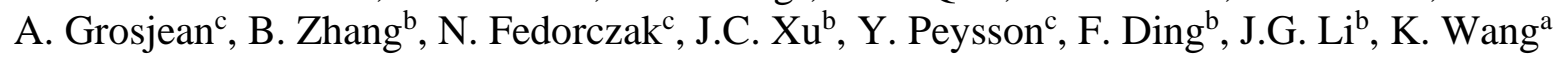

\author{
${ }^{a}$ Key Laboratory of Optoelectronic Devices and Systems, College of Physics and Optoelectronic Engineering, \\ Shenzhen University, Shenzhen 518060, China \\ ${ }^{b}$ Institute of Plasma Physics, Chinese Academy of Sciences, Hefei 230031, People's Republic of China \\ ${ }^{c}$ CEA, IRFM, F-13108 Saint Paul-Lez-Durance, France. \\ ${ }^{d}$ Max-Planck-Institut für Plasmaphysik, Boltzmannstrasse 2,D-85748 Garching, Germany \\ e Laboratorium voor Plasmafysica - Laboratoire de Physique des Plasmas, Association 'EURATOM - \\ Belgian State', Trilateral Euregio Cluster, 30 Avenue de la Renaissance, B-1000, Brussels, Belgium \\ ${ }^{f}$ Institut Jean Lamour, UMR 7198, CNRS-U. de Lorraine, F-54506 Vandoeuvre Cedex, France
}

taiyang.lin@ipp.ac.cn, zhangling@ipp.ac.cn

\begin{abstract}
.
This study uses the diversity of materials at known locations in the Experimental Advanced Superconducting Tokamak (EAST) to extract, local information on which plasmasurface interaction processes are dominant during Ion Cyclotron Resonance Heating (ICRH) (near vs far field effects). Metallic impurity content is indicated by the intensity of impurity line emissions observed with a fast-time-response extreme ultraviolet (EUV) spectrometer, normalized to line-averaged plasma density, i.e. $\mathrm{I}_{\mathrm{mp}} / \mathrm{n}_{\mathrm{e}}$. Parametric dependencies are explored over scans of ICRH and LH (Lower Hybrid) power and for different toroidal phasings between straps, strap power balance, and magnetic configurations. This diversity of behavior is interpreted as the signature of different physical processes. Before 2017, as only the upper divertor region contained tungsten (W), the $\mathrm{W}$ content in the core used to increase a lot when moving from lower (LSN) to upper single null (USN) configurations, and was correlated with the total injected power rather than the ICRH power. Molybdenum (Mo), covering the part of the inner wall facing one ICRF antenna appear sensitive to this antenna power and phasing benchmarked by modelling suggesting a probable interaction due to residual ICRF waves crossing the plasma. Materials close and magnetically-connected to an active antenna show better correlation with ICRF antenna electrical tuning than those which are far away, or not connected. This is particularly the case with W since 2018, because the limiter tiles of the LH launchers were changed from graphite to tungsten. In these latter conditions, it is shown that $\mathrm{W}$ sources at the mid-plane (equatorial plane) contribute to a significant fraction of the core contamination by tungsten ( $25 \%$ in ohmic regime, and more during ICRH).
\end{abstract}

\section{Introduction.}

The Experimental Advanced Superconducting Tokamak (EAST) prioritizes high power long pulses in steady state in support of future experiments on ITER and the China Fusion Engineering Test Reactor (CFETR). Demanding more power becomes yet challenging in terms of plasma surface interaction. Among the 12MW ICRH power available at the generator, only $2 \mathrm{MW}$ are most often used, not only because of coupling limitations but also because of excessive impurity production. This study focuses on ICRF-enhanced plasma surface interaction, the localization of main impurity sources, and the different physical mechanisms at play. Different magnetic configurations are also explored to further analyze the contribution of different divertor areas and attempt to provide some quantitative estimations. 
When the coupling efficiency of ICRF wave is low - which is the case in EAST $[1,2](<35 \%)$ - high RF near-fields are excited, leading to DC potential rectification, known for enhancing plasma-wall interactions [3-13]. This can have particularly deleterious effects near antennas where the strongest near-fields are observed, but also affects far and even non-magnetically connected regions, to which we will refer to as far-field effect [4]. Near-field effects have been extensively characterized in most devices equipped with ICRH such as Tore Supra $[4,5]$, Alcator C-Mod [6-8], JET [9], ASDEX Upgrade [10], the LineAr Plasma Device (LAPD) [11] and in EAST where preliminary results were already published [12]. An excellent overview can be found in [13]. Far-field effects, however, are rarely discussed [14-17] not only because they can occur in unexpected locations that are often poorly diagnosed - such as the high field side wall of tokamaks - but also because in well-diagnosed locations - typically objects at low field side - their intensity is usually smaller than other phenomena such as near-field effects transported along magnetic field lines. This study aims to demonstrate that a deeper understanding of how to reduce them can be critical in making ICRH compatible with long pulse operation. Major difficulty stems from the fact that most high-Z impurities, in EAST specifically, can only be seen in the core using an Extreme UltraViolet (EUV) spectrometer $[18,19]$ which main characteristics are discussed in the next section, while direct sources characterization for instance with visible spectroscopy was only available on the upper divertor. Others diagnostics such as soft X-ray [20] and bolometry are evidently available, however will not be used in the present study which the aim is to distinguish different species as they are produced in different regions. The present study highly relies on the diversity of materials in the vessel to extract relatively local information. This diversity of materials includes titanium in a location only magnetically connected to one ICRH antenna (B-port, magenta in Fig.1), and tungsten in the LH grill's four lateral limiters (since 2018, before they were graphite). While first aspects presented are focusing on the characterization of ICRH-related sources of impurities - already well-documented by past experiments in other devices [3-17] - an important goal of this study (by collecting information on different regions at the same time), is to provide new information on the rather local or global character of dominant mechanisms, with efforts to also disentangle the so-called near from far RF fields effects. Comparisons between different campaigns and magnetic configurations were exploited to extract qualitative information on impurity sources.

We first introduce the experimental protocol and setup, including a description of the EUV spectrometer, data treatment applied and the materials location in the chamber.

The third section starts by showing the importance of magnetic connections to active ICRF antennas, and further presents observations of ICRF-enhanced plasma surface interactions, in different locations and relying on different mechanisms such as far-field on non-magnetically connected objects and near-field on magnetically connected ones. Parameter like the RF phasing and power are essentially scanned to disentangle different effects.

In the last section, we compare situations with and without tungsten at the mid-plane (equatorial plane), and show that sources of impurities located around the mid-plane in surfaces magnetically connected to ICRH antennas have very deleterious influence on the core contamination due to impurity. Further details on different aspects mentioned along the paper are provided in the appendix. 


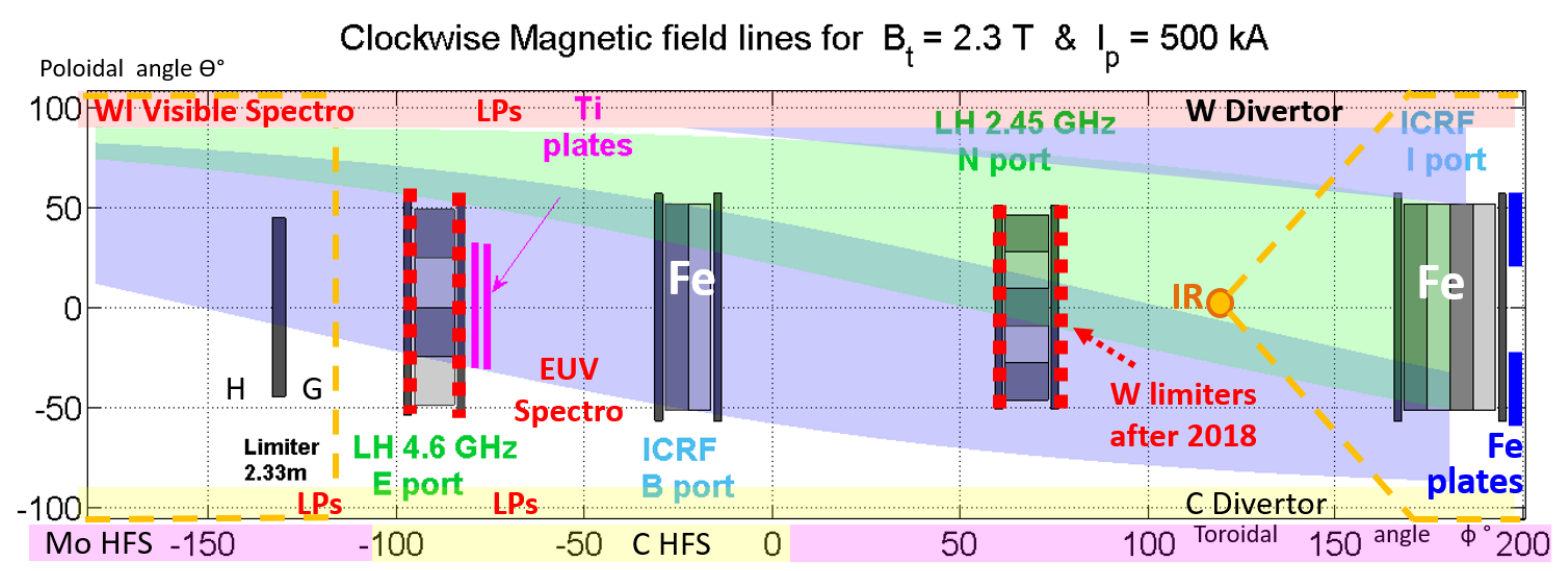

Figure 1. Toroidal-Poloidal ( $\phi-\Theta)$ 2D map of EAST showing magnetic connections between objects, materials, heating systems and EUV spectrometer, seen from center of the torus with one half of high field side wall in Mo and the other half in graphite. Unlike in [12], limiters of the LH launchers (red dots) will in the last section (after 2018) be changed from graphite into massive tungsten.

\section{Experimental conditions and protocol in EAST.}

EAST has two divertors allowing to perform discharges in double (DN), lower (LSN) and upper single null (USN) configurations. In this study, discharges in USN configurations will mostly be used, and some will be briefly compared with similar discharges in LSN to allow quantitative estimations. Almost all discharges presented in the study were typically using the following set of parameters: toroidal magnetic field $\mathrm{Bt}=2.3 \mathrm{~T}$, plasma current $\mathrm{Ip}=500 \mathrm{kA}$, major radius $\mathrm{Ro}=1.85 \mathrm{~m}$, minor radius $\mathrm{a}=0.44 \mathrm{~m}$, triangularity $\delta=0.5$ and elongation $\kappa=1.65$. Daily lithium coating ( 1 gram) is used to keep low-Z impurities like oxygen and carbon down to acceptable levels in terms of radiated power. A consequence is also to keep the hydrogen concentration almost always below 5\%, favorable for minority heating. In addition, lithium coating is a great tool to mitigate high-Z impurity contamination of core plasma [21].

This mitigation decreases during the experiments as $\mathrm{Li}$ is eroded and redeposited elsewhere. In this study all plasmas were in L-mode and USN configurations. Key auxiliary heating systems for our study are Lower Hybrid Current Drive (LHCD) and ICRH. EAST is also equipped of two neutral beam injectors and one $170 \mathrm{GHz}$ electron cyclotron Gaussian beam launcher that will only be used on some cases in the last section of this paper. Lower Hybrid is almost always used for driving current and helps doing steady state discharges. The two launchers are located in the E-port $\left(4.6 \mathrm{GHz}\right.$ and $\left.1.79<\mathrm{n}_{\|}<2.26\right)$ and $\mathrm{N}$-port $(2.45 \mathrm{GHz}$ and $\left.1.85<\mathrm{n}_{\|}<2.6\right)$ [22]. Only in the last section will we present data obtained after the LH launchers' lateral protections were upgraded from graphite to tungsten. The two ICRH antennas are heating hydrogen minority in deuterium plasmas; one is located in the B-port $(31.5 \mathrm{MHz})$ and composed of two arrays of two straps and the other is located in the I-port (35 MHz) and composed of a toroidal array of four straps (Fig.1 and Fig.A3) [1, 3, 23]. The power lost in the transmission line is not measured experimentally, however calculation of the RF losses show that they are always less than $15 \%$, resulting from uncertainties on power estimations detailed in [24]. In addition, the phase between straps cannot be changed in real time [25]. The different phase and matching are therefore set in the vacuum and the same settings are maintained with the plasma.

When not specified, all straps in a given antenna were fed with RF currents of comparable magnitude in dipole phasing $\left(180^{\circ}\right.$ phase difference between RF-currents in toroidally-adjacent straps).

In EAST, a fast-time-response flat-field extreme ultraviolet (EUV) spectrometer working at 20-500 $\AA$ called "EUV_Long" is used to observe the line emissions from high-Z impurity ions. The detector of charge-coupled device (CCD) could be moved along the focal plane by a remotely controlled pulse motor to scan the wavelength range to be observed. In 
order to monitor Ti XVIII (Ti $\left.{ }^{17+}\right) 144.759 \AA$, Fe XXIII $\left(\mathrm{Fe}^{22+}\right) 132.91 \AA$, Mo XXXII $\left(\mathrm{Mo}^{31+}\right)$ 127.868 $\AA$, W-UTA (tungsten unresolved transition array noted $\mathrm{W}$ for simplifying) at 45-70 simultaneously, the wavelength range is fixed to be $20-150 \AA$. The CCD $\left(26.6 \times 6.6 \mathrm{~mm}^{2}\right.$ with $1024 \times 255$ pixels) is usually operated at $5 \mathrm{~ms} /$ frame with full vertical binning (FVB) mode. The spectral resolution of the spectrometer is $0.22 \AA$ at $100 \AA$. The line of sight (LOS) crosses central plasma as shown by the red dotted lines in Fig.2. One may find detailed information of the spectrometer in Ref. [18,19]. Lines intensity are systematically normalized by lineaveraged electron density to indicate impurity content. Benefiting from good reproducibility of the discharges along experimental sessions, transport effects will be considered constants and variations will rather be interpreted as a change in the sources of impurities. This assumption makes sense for most species radiating close to the edge (Fig.2) - where transport is essentially diffusive and turbulent in L-mode [26] - and easily penetrate inside the plasma [27]. The edge turbulence - and therefore transport - known as blobs or avaloids is actually expected to be reduced by the application of ICRH as shown in [28], while analogous study will be presented in a dedicated paper. It was in addition shown by controlling the injection of impurity such as nitrogen in Alcator C-Mod [29] and in JET and ASDEX-Upgrade [13, 30], that the ICRF fields do not affect the relative levels of nitrogen in the plasma significantly, such that the turbulent transport in the plasma edge appears usually insensitive to the application of ICRH. However, we acknowledge this can be questionable for $\mathrm{W}$ coming from the divertor region, as it is not only expected to be better screened [27], but also its emissivity mainly comes from the core plasma (Fig.2), therefore making it sensitive not only to transport in the edge but also in the central region, generally convective neoclassical. Some changes may indeed occur, yet as temperature and density profiles remained fairly similar along the experiments, this transport is not expected to significantly change our conclusions. More importantly, $\mathrm{T}_{\mathrm{e}}$ in the core did not change significantly $(<10 \%$ as shown in Fig.3f) and its profile remained relatively flat at the center without fast particles. Note this is substantially different from nitrogen experiments in JET where the deposition of the ICRF power at the center can on the contrary help prevent metallic impurity from accumulating in the core [31]. It is however important to point out, not only were experiments in JET conducted in H-mode, but also that this beneficial effect occurs above a power threshold of approximately $3 \mathrm{MW}$, which is beyond what can be done in EAST. Moreover, the spectrometer was measuring a W continuum (W-UTA) which together with the profiles all having fairly constant shapes, guarantee that $\mathrm{W}$ intensity measured by the EUV spectrometer was not significantly influenced by $\mathrm{T}_{\mathrm{e}}$ changes and was fairly representative of the sources. This assumption will nevertheless be benchmarked by neutral tungsten (WI) visible spectroscopy, with 16 lines of sight covering both the inner and the outer targets of the upper divertor at $150 \mathrm{~Hz}$ sampling rate. 


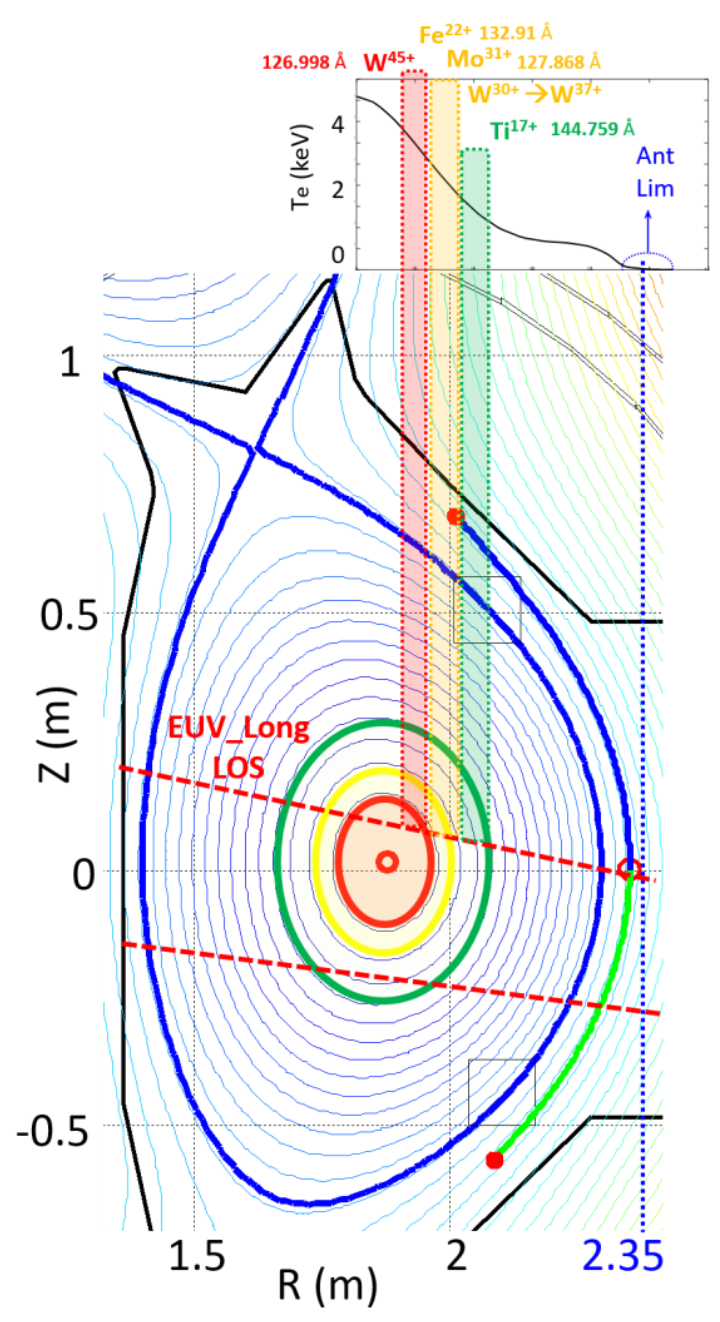

Figure 2. Spectral lines observed by EUV spectrometer in the present study with the corresponding emitted wavelength $\lambda$ and temperature $T_{e}$ at which the ionization of the given state is approximately maximal

In complement of the results presented in [12], we will now follow other lines such as $\mathrm{Fe}^{22+}$ $(132.91 \AA)$ and $\mathrm{Mo}^{31+}(127.868 \AA)$, corresponding to the different materials summarized in the table at the top of Fig.2. The presence of these species will then be interpreted as the footprints of plasma-material interaction in different locations summarized in Table 1:

- Iron $(\mathrm{Fe})$ is essentially found on antenna Faraday screens (underneath a boron carbide coating). Later, two silver coated plates were also fixed on the right side of the I-port antenna, and we will see in section 3.3 that these plates partially melted

- Molybdenum (Mo) covers two third of the high field side wall facing the I-port antenna $\left(0^{\circ}<\phi<250^{\circ}\right.$ and $-90^{\circ}<\Theta<90^{\circ}$ as indicated by pink regions on the bottom of Fig.1)

- Graphite is found on all antenna limiters (before 2018), in the lower divertor and on a third of the high field side wall

- Tungsten (W) is only found in the upper divertor (before 2018). Rather than looking at high ionized states often sensitive to small temperature variations, we will be following the evolution of the tungsten continuum (W-UTA noted W for simplifying) which is composed of emission lines from $\mathrm{W}^{24+}$ up to $\mathrm{W}^{45+}$ depending on $\mathrm{T}_{\mathrm{e}}$.

- $\quad$ Titanium noted $\mathrm{Ti}$ for $\mathrm{Ti}^{17+}(144.759 \AA$ ) passive plates were installed to serve as impurity tracers of interactions regions magnetically connected to an ICRF antenna. Plates were fixed one toroidal location of the outer mid-plane $\left(\phi \sim-90^{\circ}\right.$ and $-30^{\circ}<$ $\Theta<30^{\circ}$ ) magnetically connected only to the B-port as shown by the blue region and magenta vertical lines in Fig.1. Ti was the best candidate mainly because an 
emission line can easily be identified from the spectra observed with EUV spectrometer without a blend with others species present in EAST, but also because it can be used as a permanent plasma facing component without affecting the vacuum conditions and that its cost is reasonably low.

\begin{tabular}{|c|l|}
\hline Materials & \multicolumn{1}{|c|}{ Location } \\
\hline $\mathbf{C}$ & Limiters, Lower divertor and a third of the high field side wall \\
\hline $\mathbf{F e}$ & ICRH antennas Faraday screens \\
\hline $\mathbf{T i}$ & Between D and E port Mag. Connected to B-port \\
\hline $\mathbf{M o}$ & All walls except $130^{\circ}$ of the high field side facing B-port \\
\hline \multirow{2}{*}{$\mathbf{W}$} & Before 2018: Only upper divertor \\
\cline { 2 - 3 } & After 2018: Upper divertor and LH launchers limiters \\
\hline
\end{tabular}

Table 1. Summary of materials location in EAST

\section{Links between ICRH and impurities production}

By powering each antenna successively (B-port then I-port), we show in Fig.3 how the different species characteristics of different location behave according to their position in EAST. In particular we can observe the importance of magnetic connections between each species and active ICRH antennas. In between both ICRF pulses (from 5 to 6s) the plasma is only heated with 1.4MW LH power, which sets a reference level for each impurity without ICRH. ICRH antennas were powered in dipole phasing and with $5 \mathrm{~Hz}$ modulations of power between 0.5 and $1 \mathrm{MW}$ as shown in Fig.3e. The electron temperature and density remained fairly constant all along the discharge as represented on Fig.3f. The same graph shows the radiation power from bolometry which increases with ICRH as well as the Soft X-ray [20] central chord (Fig.3h), while plasma energy content (Fig.3g) does not change much, suggesting either that ICRH power is not well coupled or not well absorbed. D $\alpha$ signal is also provided in Fig.3g, showing the rather steady level of turbulence typical of L-mode discharges in EAST.

All metal impurity line intensities increase fairly similarly during application of LH power. It is worth mentioning that, seen from our EUV spectrometer [18], the $\mathrm{Mo}^{31+}$ line is hard to distinguish compared to other species, such that its temporal evolution is not always easy to interpret. When the ICRH antennas are activated, Ti and Fe contents in the plasma increase and correlate strongly with power modulations visible on both signals. As Fe and Ti-covered objects are both magnetically connected to the B-port antenna, the strongest interactions occurred when this antenna was active. Fe is also well connected to the I-port antenna, so we expect similar levels in both cases, but since the B-port antenna is much smaller, higher power densities are expected for same antenna loading and power. However, Mo showed opposite behavior and higher content was observed when the I-port antenna was powered. Since Mo is symmetrically present all around the vessel's low field side, we believe it does not come from there, otherwise it should follow a similar trend as Fe. As represented on Fig.1 by the pinkish region, the I-port faces Mo high field side wall whereas the B-port faces carbon. Impurities variations over an experimental phase scan of I-port antenna hints that Mo might come from the high field side wall facing the I-port. This will be presented in the next section together with modelling results supporting this claim. In the next subsection, we show that W originating from the divertor region seems to react equally to ICRH and LH power. We show that the RF rectification on divertor targets plays a minor role on impurity productions. Launched ICRH power can either be lost at the edge, or absorbed by the plasma and increase fluxes on divertor. A discussion on ICRF waves' absorption efficiency is therefore added and a correlation with total injected power is found. Finally, the last subsection discusses nearfields effects on surfaces magnetically connected to active antennas. 


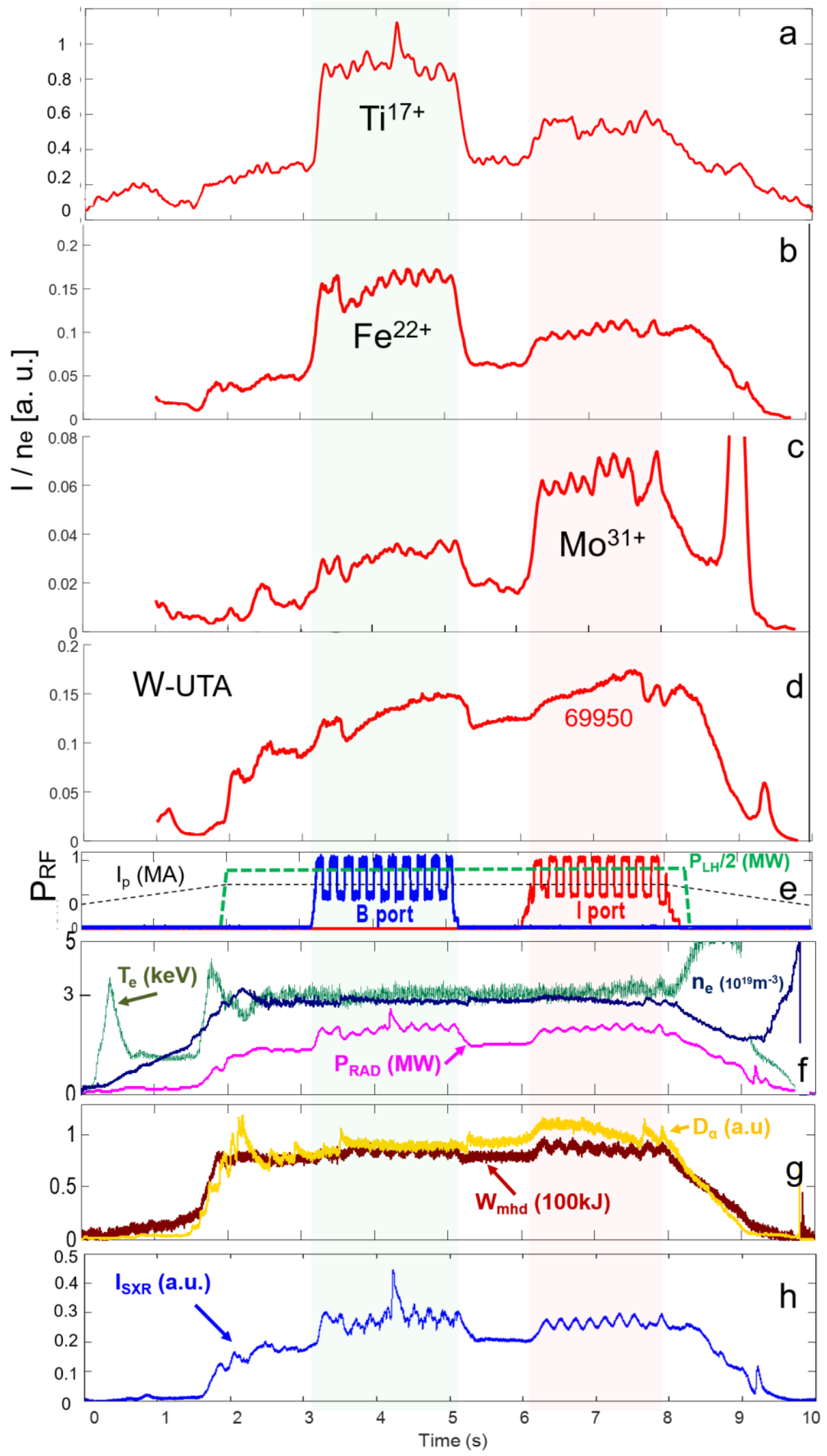

Figure 3. Time evolution of line-averaged electron density normalized line intensity of (a) Ti XVIII at

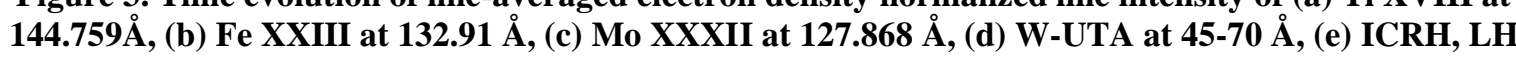
powers and plasma current, (f) radiated power from bolometry, electrons temperature from ECE and density from interferometry, $(g)$ plasma energy $\left(W_{\text {mhd }}\right)$ and $D \alpha$ and $(h)$ central chord of Soft X-ray for EAST \#69950 discharge. Adapted from [12]. 
From Fig.3, we know that the total ICRH power $\left(\mathrm{P}_{\text {ICRH }}=\mathrm{P}_{\mathrm{I}}+\mathrm{P}_{\mathrm{B}}\right)$ is not itself a fully relevant parameter to estimate impurity levels as a function of the power from $\mathrm{I}\left(\mathrm{P}_{\mathrm{I}}\right)$ and $\mathrm{B}$ ports $\left(\mathrm{P}_{\mathrm{B}}\right)$ have different influence on different impurities. It consequently matters to have a relatively constant ratio $\mathrm{P}_{\mathrm{I}} / \mathrm{P}_{\mathrm{B}}$ over power scan, which is kept close to unity $\left(\mathrm{P}_{\mathrm{F}} \approx \mathrm{P}_{\mathrm{B}}\right)$. Since plasma shape did not change much, EUV spectral line intensities were systematically normalized to lineaveraged electron density from interferometer and averaged over small periods of time (between 0.02 and 0.1s) and noted Imp. For each species and discharge, ICRH-related impurity production $Y_{\text {shot }}$ is calculated as:

$$
Y_{\text {shot }}=\left(I m p_{\text {withIC }}-\operatorname{Im} p_{\text {withoutIC }}\right) / \max \left(I m p_{\text {withIC }}-\operatorname{Im} p_{\text {withoutIC }}\right)_{\text {overall shots }}
$$

\subsection{Far-field effects on inner wall}

The high field side wall is a surface almost never magnetically connected to active ICRH antennas at the low field side. Interactions at the high field side are therefore in principle not correlated to what happens at the low field side as seen on Mo behavior in Fig. 3. A way ICRH may have an impact on the inner wall is through the far-field, often resulting from a poor absorption efficiency leading to residual ICRH power crossing the plasma. By changing the phase difference between RF currents on toroidally adjacent straps of an ICRH antenna, the launched spectrum changes together with wave propagation and absorption properties. This allows discussing ICRH far-fields effects on the inner wall. In EAST, only phases of the I-port antenna were changed whereas the B-port antenna always operated in presumably dipole phasing. A series of discharges with similar power and loadings on both antennas and different phasing of adjacent straps of the I-port antenna were studied and results are shown in Fig.4. Ti, Fe and $\mathrm{W}$ remained relatively insensitive to the phase variation, which is surprising as both modelling (see appendix) and results from other devices respectively predict higher fields and an increase in impurity production as phasing tends towards monopole [1, 11,33]. On the one hand the absence of real time feedback control of EAST ICRH port phasing [25] can be responsible for this, but on the other hand, Mo content varied in agreement with what is expected from observations in other devices, namely an increase in Mo production going from dipole to monopole. Once again, this peculiar observation leads us to consider the high field side wall as a likely source of Mo correlated with the I-port antenna settings.

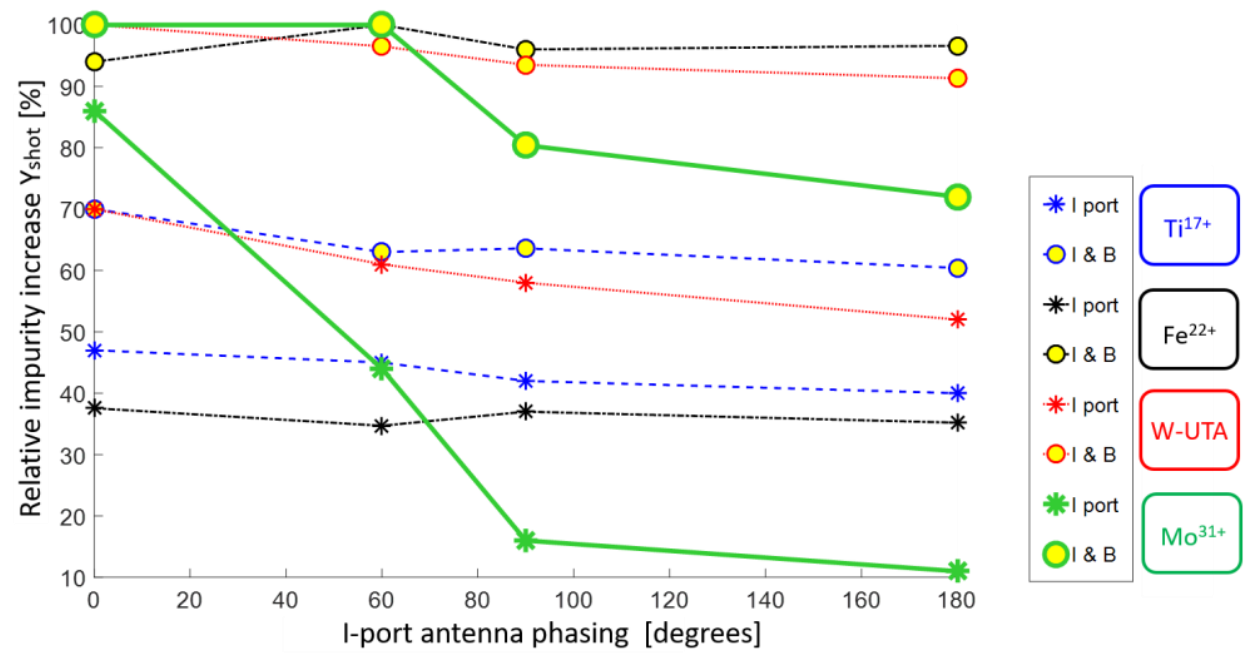

Figure 4. ICRH-related impurity production increase $\left(Y_{\text {shot }}\right)$ as a function of the I-port antenna phasing

In addition to the I-port antenna which phase is being scanned, yellow circles provide additional information on the relative contribution to impurity contents of the B-port antenna which is being powered with constant dipole phasing with same power. Each impurity content therefore increases and the dependence on I-port phasing is diluted. When both antennas are 
powered in dipole phasing, it appears that the B-port produces more of each impurity than the I-port (except tungsten from the divertor region which will be further discussed in the next section). In the case of Mo, it is interesting to notice that almost all is being produced by the B-port when both antennas are excited with dipole phasing, while the majority ends up being produced by the I-port when its phasing drops towards monopole. For similar power and phasing, the fact that the B-port is the principal cause of impurity production can be partially understood considering its size, twice smaller than the I-port antenna, leading to much higher power densities for similar generator power and loadings. This is particularly visible for the $\mathrm{Fe}$ characteristic of near-field interactions, whose level is quite low all over the I-port phase scan and increases by $150 \%$ when the B-port is activated.

Knowing this, and that Mo covers all low field side walls, if it were to originate from the low field side, it would likely behave like Fe. Mo is however sensitive to I-port phasing, suggesting that it may come from the high field side wall facing I-port. Since dedicated experiments to account for the origin of Mo are too challenging due to the lack of appropriate diagnostic, wave absorption was first assessed with TOMCAT 1D code [34] (described in appendix A1), as it requires minimal resources and is therefore useful for preliminary computations. Inputs were basically the toroidal wave number of I-port antenna calculated separately [1] and analytical profiles analogous to experimentally measured temperature and density profiles along reference discharge (69950). Complementary to these 1D simulations, wave propagation was also simulated in 3D with EVE code [35] (description in appendix A1). Here the magnetic equilibrium was also used, and antenna spectra computed based on the toroidal phasing between straps of the I-port antenna (Fig.A3b). Since simulations with both codes are essentially made in the core, with edge densities high enough to support Ion Bernstein waves propagation and avoid including the lower hybrid resonance in the domain of simulation, we acknowledge that these results and in particular the parallel components of the electric field cannot be straightforwardly attributed to the slow wave. TOMCAT predicts a degradation of the single pass absorption from $50 \%$ in dipole phasing $\left(\phi=180^{\circ}\right)$ down to $15 \%$ in monopole phasing $\left(\phi=0^{\circ}\right)$ with an increase of the parallel component of the electric field on the high field side (Fig.5). Fig.6 shows the real part of the iso-contours of the left-handed polarized electric field computed in 3D by EVE code, while complementary results are provided in appendix 1.

First, we can see in the plasma center that as phasing gets closer to zero, the fast wave is converted into an ion Bernstein wave, which may not be optimal for the efficiency of the wave absorption in the core plasma. This observation can be interpreted as consistent with the experiments were the absorption efficiency also drops as phasing shifts away from dipole down to monopole. It is yet important to stress that the modeling of the Ion Bernstein wave with only a second order expansion like in EVE, may be subject to discussion away from the resonance. Still, given that the agreement with TOMCAT - which relies on a Finite Larmor Radius assumption of the Kennel-Engelmann operator up to $\mathrm{N}=3$ harmonic heating rather than of the dielectric tensor - is pretty good, we are confident that these results are a priori still relevant.

Secondly, closer to the high field side, the fast wave in principle can also convert into slow wave, which mechanism is usually associated with RF sheath excitation and enhancement of plasma surface interactions. Unfortunately, to accurately model this effect, both EVE and TOMCAT would require extending their simulation domains to the vessel wall including at least a cold, but ideally hot plasma description, which is not the case. Consequently, when phasing shifts away from dipole, despite the parallel component of the electric field $\left(\mathrm{E}_{\|}\right)$ increases on the high field side as shown in Fig.5, this cannot be attributed to the slow wave. Accurately reproducing the same exercise with EVE (Fig.A1a), proved to be challenging since it does not include an accurate model for edge damping processes at this stage. As a result, it is always assumed that the total $4.6 \mathrm{MW}$ are absorbed in the plasma core regardless of the toroidal phasing and absorption efficiency. For better estimation, we could for instance scale the power absorbed in EVE with the single pass absorptions modelled with TOMCAT, but this would still be inaccurate since the remaining unabsorbed power is not necessarily lost in the edge. Again, alleviating this assumption would require extending EVE up to the vessel wall, 
as was explored e.g. in [37], and is left for future work. Even then, no diagnostic allowing to measure density profiles near the high field side of EAST, accurate modelling of region with densities bellow the lower hybrid resonance would be very challenging, not only numerically due to short wavelength, but also to provide accurate inputs. Note these results would be fairly relevant if the FW cutoff layer is close to the wall, which regrettably again, no available diagnostic can measure.

Still, assuming that the vessel behaves as a Faraday cage from which nothing can leak, since the absorption efficiency is better in dipole than monopole phasing, we expect edge effects to be more important in the latter case. In addition, not only materials present in the low field side - such as iron from antenna Faraday Screen - are fairly insensitive to the I-port antenna phasing (black curves in Fig.4), but also modelling result point in the direction of higher fields on the high field side. Insofar, we believe plasma surface interaction on the Mo surfaces on the inner wall do matter when the facing I-port antenna is powered in monopole phasing.

The behavior of the carbon from the high field side wall facing B-port unfortunately does not show any clear correlation with the B-port antenna since it can also be found on all antenna limiters. So-called parallel, positive and negative field polarizations are also available in appendix 1 (Fig. A1) to better exhibit mode conversion nearby resonance layer $(\mathrm{R}=1.7 \mathrm{~m})$. It can also be seen on loaded spectra in Fig. A2b that less than $60^{\circ}$ phasing leads to the excitation of low $\boldsymbol{k}_{\|}$modes which are poorly absorbed and can result in proper modes in the plasma. These modes propagate in plasma edge and can lead to power losses and further enhance plasma surface interactions $[33,38]$.
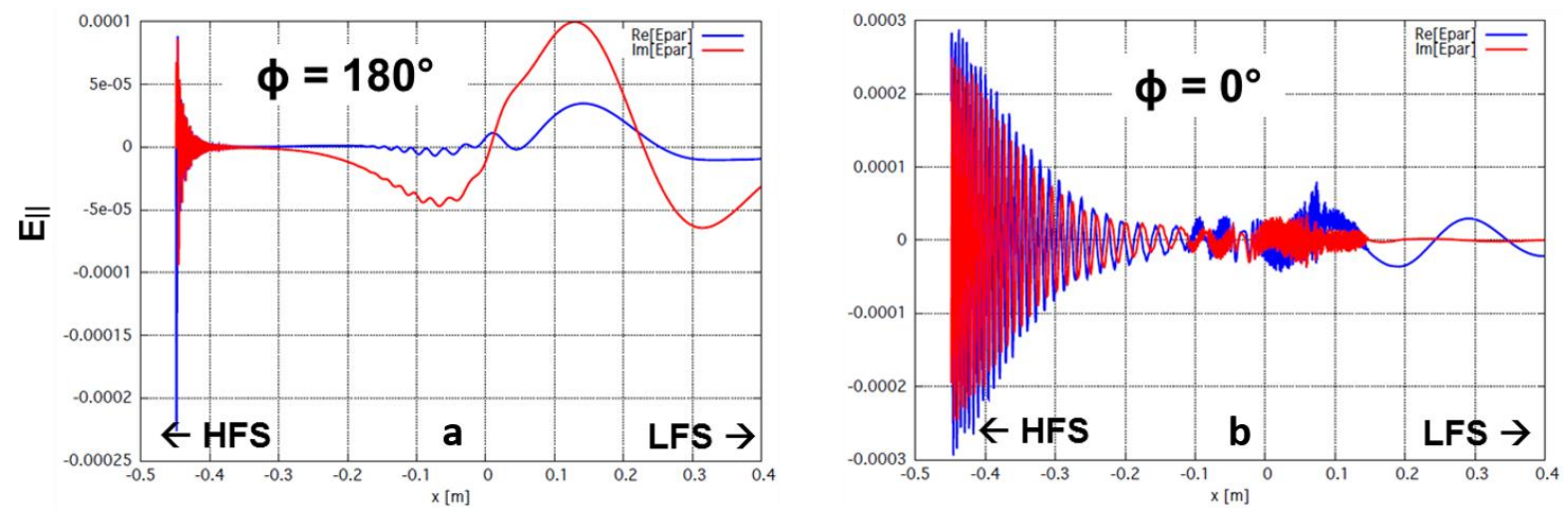

Figure 5. Real and imaginary parts of the parallel component of the electric field computed with TOMCAT code for $(a)$ dipole $\left(180^{\circ}\right)$ and $(b)$ monopole $\left(0^{\circ}\right)$ phasing of the I-port ICRF antenna adapted from [36]
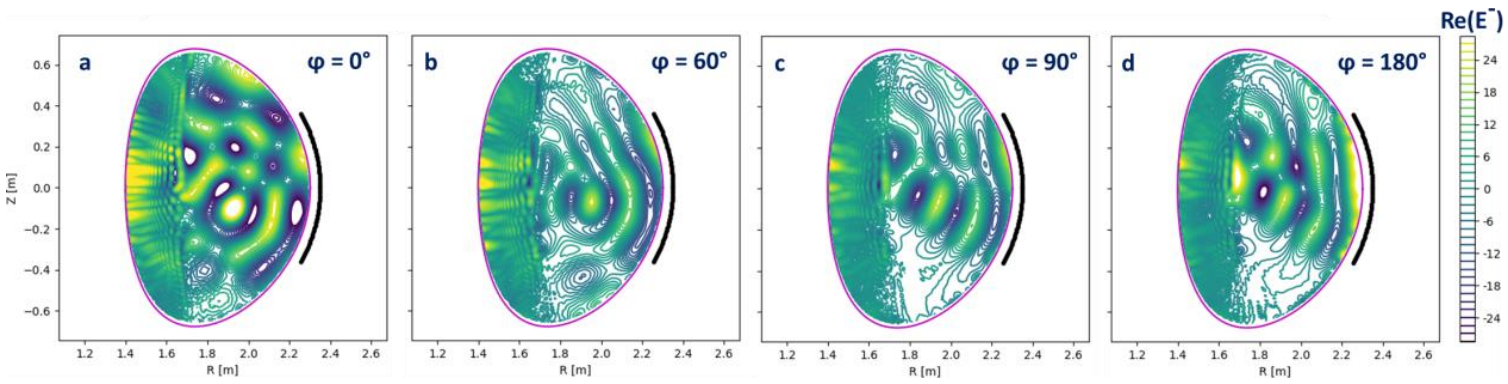

Figure 6. Iso-contours of the left-handed polarized electric field (real part), in $\mathrm{kV} / \mathrm{m}$, for poloidal cuts taken at the toroidal location of the antenna, computed with EVE code and assuming 4.6MW of total absorbed RF power for various phasings of the I-port ICRH antenna:

(a) $\varphi=0^{\circ}$, (b) $\varphi=60^{\circ}$,(c) $\varphi=90^{\circ}$,(d) $\varphi=180^{\circ}$ 
We can also look how Mo behaves while changing ICRH power and keep phasing as close as possible to dipole on both antennas. Three series of discharges with scan of PICRH at the generator in different conditions were analyzed. $Y_{\text {discharge }}$ as defined in eq. 1 is represented in Fig.7 for Mo. In the blue series only PICRH increased, while in the black series PICRH increased together with $\mathrm{P}_{\mathrm{LH}}$. In the red series, power balance on the B-port antenna straps is changed over the scan: one of the four radiating straps is typically switched off as the power is increased on the other straps. Through black and blue series in Fig.7, Mo seems to have no clear correlation with the ICRH power. The red series for which the power is unbalanced on the B-port antenna straps confirms the negligible impact of B-port antenna and its settings on Mo, consistent with a source at the inner wall facing I-port.

Note, decreasing phasing closer to zero not only has consequences on far-fields but also on near-fields as shown in Fig.7 of [23]. Near-fields effects will be discussed in further details in section 3.3.

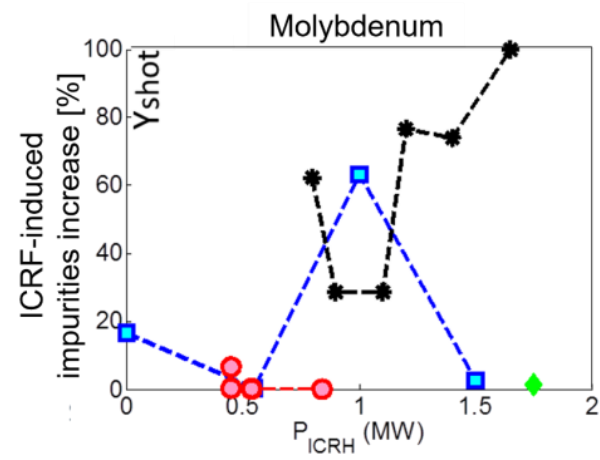

Figure 7. ICRH-related Mo production $\left(Y_{\text {shot }}\right)$ as a function of ICRH power. In the blue series, LH power remained constant while it changed in the black series. Power balance on straps was changed in the red series. Green dot corresponds to a discharge only heated with ICRH power (balanced on all straps).

\subsection{Divertor region, a combination of all effects}

In this section, we will only discuss cases before 2018, when W could only be found in the upper divertor. We will therefore interpret W-UTA from the EUV spectrometer as a modification of $\mathrm{W}$ source assuming transport does not have a strong impact on core $\mathrm{W}$ content. In addition, the spectral shape of the $\mathrm{W}$ continuum was constant for the different discharges and RF power, i.e. the ionization remained the same in every condition. $\mathrm{Y}_{\text {discharge }}$ as defined in eq. 1 is represented in Fig.8a for W.

For constant LH power, we observe similar W content when the power is unbalanced (red series) than when it is well balanced (blue series). This suggests that the divertor region is unresponsive to the antenna settings and only depends on the power injected. Furthermore, looking at the cases when ICRH increases together with LH power (black series, along which the current drive efficiency did not change significantly, $\mathrm{V}_{\text {loop }} \sim 0.1 \pm 0.02 \mathrm{~V}$ ), W content increases strongly indicating that the divertor region is sensitive to the total power injected rather than ICRH power alone.

This can be confirmed by tracing impurity increase as function of $\mathrm{P}_{\text {TOT }}$ for all discharges (Fig.8b). The impurity increase is defined as $\mid$ Imp during IC -Imp before IC $\mid$ with ICRH (black stars and red circles) otherwise $\mid \operatorname{Imp}$ during $L H-\operatorname{Imp}$ before $L H \mid$ (magenta series). Note, the linear trend of both black and red dots, meaning that $\mathrm{W}$ production increases in similar fashion with LH and ICRH. 

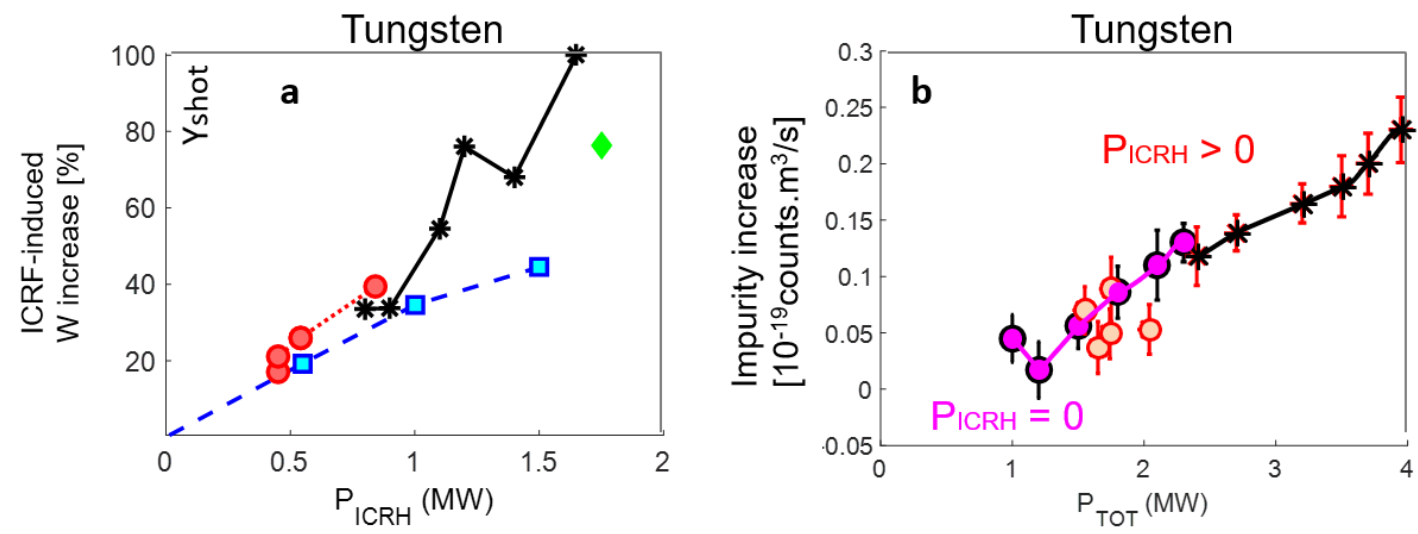

Figure 8. (a) $\mathrm{W}$ production ( $Y_{\text {shot }}$ as defined in eq.1) as a function of ICRH power and (b) W increase normalized by electron density against total power. Blue, red and black series in graph a correspond to the same discharges as in Fig.7. Graphs b also represent the same discharges except these of the blue series, in addition points without ICRH (magenta series) were added

(adapted from [12]).

Considering how linear the red and blue trends are in Fig.8a, we should now look after footprints of non-linear RF sheath through potential rectification in the divertor region. This is provided in Fig.9, representing floating potential and ion saturation current profiles along upper divertor targets measured by Langmuir probes [39] along the black series power scan. In the case without ICRH (black curve), modification of floating potential $\left(\mathrm{V}_{\mathrm{f}}\right)$ and particle fluxes $\left(\mathrm{J}_{\text {sat }}\right)$ are weak, but when ICRH is activated (colored curves) rectification is significantly stronger and increases together with ICRH power. Along the power scan, the inner strike point shifted from a probe onto another, explaining differences in some inner probes' signals. This stands as a footprint of the effect of ICRH transported along field lines up to the divertor, and its localization is furthermore consistent with others dedicated studies [40, 41]. It is interesting to note that the potential seems to increase linearly with the ICRF power. Similarly, as shown by the red and blue series in Fig.8a, the tungsten production in the divertor region also increases linearly with the ICRF power. The non-linear behavior inherent to RF-sheath is therefore completely absent in the divertor region, where interactions scale better with the total power injected (Fig.8b) than with the ICRF power (Fig.8a).

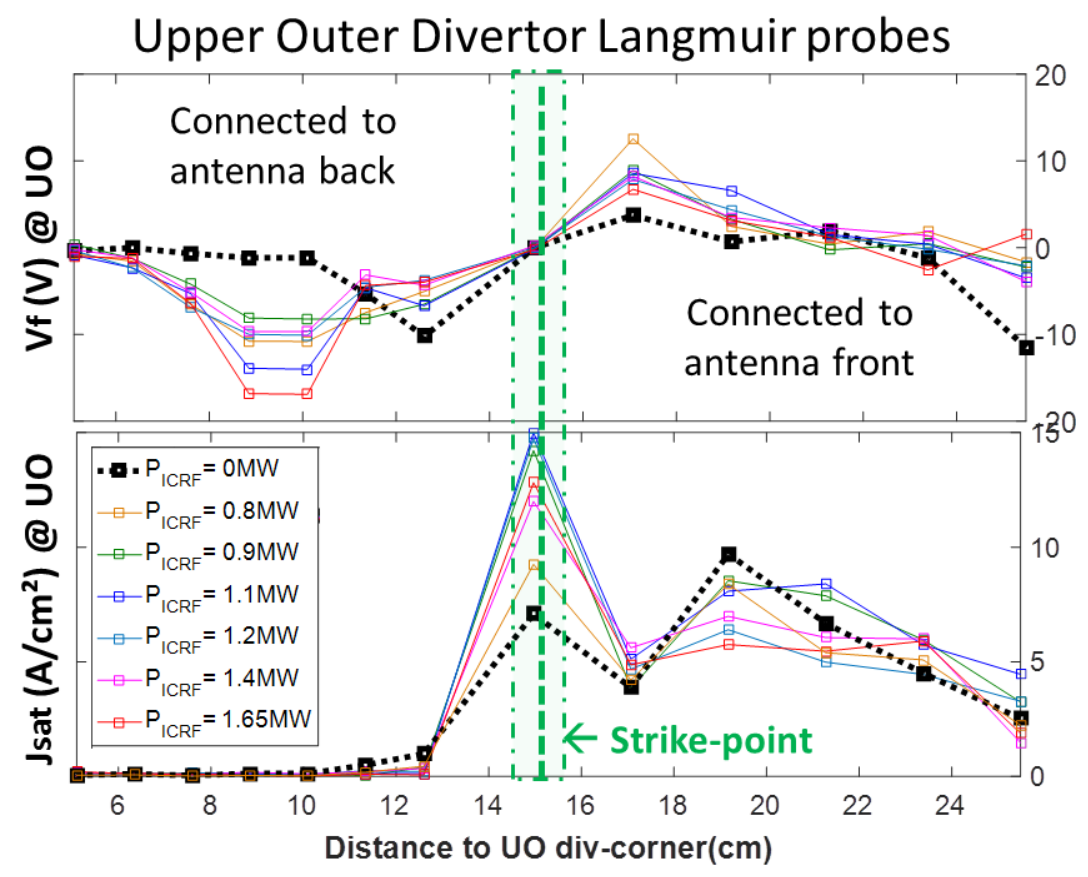

Figure 9. Floating potential $V_{f}$ and ion saturation currents $\mathbf{J}_{\text {sat }}$ profiles from outer target of the upper divertor for discharges of the black series in Fig. 8, 10 and 13. 
If we assume that the energy absorbed by the plasma ends up on the divertor targets, this can be interpreted as difficulties either to couple waves to the plasma or to have them absorbed efficiently. Since coupling efficiency remained fairly stable along the experiment, observation made in the divertor region may show some correlation with ICRF absorption efficiency, estimated based on the break-in-slope method [42]. This method consists of fitting plasma energy $W_{E N G}$ increase when applying ICRH by an exponential, providing the confinement time $\tau_{\mathrm{e}}$ :

$$
W_{E N G}(t)=W_{E N G}^{\text {beforeICRF }}-\left(W_{E N G}^{\text {beforeICRF }}-W_{E N G}^{\text {afterICRF }}\right)\left(1-\exp \left(-t / \tau_{e}\right)\right)
$$

$W_{E N G}$ is measured by diamagnetic probes and its variations are often slightly delayed compared to the ICRH power application, which was taken into account for each fit.

The absorption efficiency is finally calculated as follows:

$$
\eta_{I C R H}^{\text {Abs }}=\left(\frac{\Delta W_{E N G}}{\tau_{e}}-\frac{\Delta V_{\text {loop }}}{I_{p}}+\Delta P_{R A D}\right) /\left(\alpha P_{I C R H}^{i n j}-\frac{P_{I C R H}^{\text {refl }}}{\alpha}-\Delta P_{R A D}\right)
$$

With $\Delta$ meaning the difference between times with and without ICRH, and $\alpha$ the ratio of ICRH power lost in the transmission lines between the antennas and the voltage probes providing incident and reflected powers. The denominator basically represents the effective power step when applying ICRH. Beyond the fact that these estimations are based on single power steps - not optimal for absorption efficiency estimations - their main weaknesses come from uncertainties in power losses in transmission lines. Calculations based on the method described under section 6 of [39] result in losses always less than 15\%, such that the coefficient $\alpha$ may vary between 10 and $15 \%$. For our calculations in L-mode, we assume the most optimistic case of only $10 \%$ losses $(\alpha=0.9)$. We will anyways not discuss quantitative results but rather look at qualitative trends. This exercise was made for each discharge of the three series introduced in the previous section. Ratios of absorbed power against total power injected

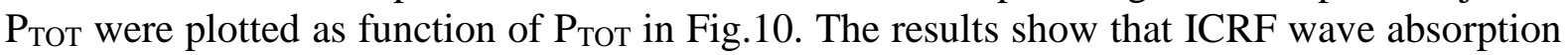
efficiency improves with total power injected. In the present regime of operation, since relatively little power is injected, ICRF absorption efficiency is often poor compared to those of lower hybrid. In addition, larger power fraction is radiated during ICRH than during LH (generally above 70\% [19]). As a conclusion, (i) the divertor target is mostly sensitive to the conducted power, and (ii) the power conducted by LH is often much higher than the one conducted by ICRH, therefore (iii) divertor region is well-correlated to total injected power (mostly LH) rather than ICRH. If the total injected power could be significantly increased, we would expect better ICRF absorption efficiency, more conducted power and therefore an increase of $\mathrm{W}$ production in the divertor region. Note for different heating conditions represented by each series in Fig. 10, absorption does not seem to improve at the same rate, yet the lack of statistics does not allow to make any strong conclusions. 


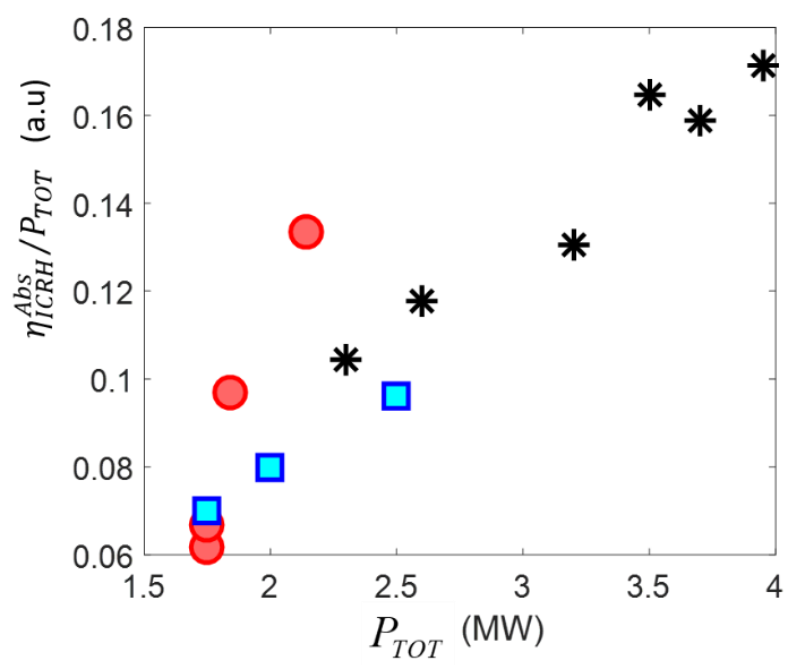

Figure 10. ICRF waves' absorption efficiency (as defined in eq. 3) normalized by total power injected as a function of total power injected

To finally confirm that the divertor region is only sensitive to the amount of power injected regardless of how it may have been launched, we propose to compare floating potential changes observed by divertor Langmuir probes [40] (Fig.11) and W sources from one visible spectroscopy line of sight (Fig.12) in both USN and LSN configurations. Fig.11 shows time evolutions of floating potential profiles along outer targets of both upper (W) and lower (C) divertors during an LSN discharge partially heated with ICRH. We see that ICRH-related potential rectification occurs in very similar fashion at the upper and lower targets. Furthermore, noticing that floating potential measured at the lower divertor are comparable to those obtained at the upper divertor (Fig.11), RF rectification in divertor targets does not seem to depend on the magnetic configuration but rather on magnetic connections to antenna front face as stressed in [42]. Based on this observation, if RF rectification played a key role in EAST divertors erosion, at least for ICRH dominated discharges, we would expect to get comparable sputtering yields in both configurations. However, as shown by WI line brightness (neutral W visible spectroscopy) for two discharges in similar conditions but different configurations (Fig.12), the upper divertor erosion rate is much higher in USN than LSN configuration. It is as if the erosion is a result of particle fluxes rather than a change in their effective sputtering yields. Furthermore, Fig.9 illustrates that the maximum of potential measured is not observed at the same position as particle fluxes (ions saturation currents), such that if we roughly estimate that the erosion results from the product of both, it may fortunately never reach deleterious regimes as both parameters reach their highest values at different position. Unfortunately, this cannot be fully confirmed as we would not only need to monitor the particles flux (deuterium + impurities), but also have absolutely calibrated lines of sight in order to estimate particle fluxes from measured photon fluxes. In the future (after 2020) EAST should operate with two tungsten divertors, it will then be of particular interest to reproduce similar discharges to push the comparison further and maybe by then also have absolutely calibrated visible spectroscopy lines of sight on both divertor targets.

As far as EAST and relatively low ICRH power are concerned, these observations suggest that $\mathrm{RF}$ rectification does not play a strong role in the erosion region of the divertor which is more sensitive to the total conducted power and of course magnetic configuration [42]. 


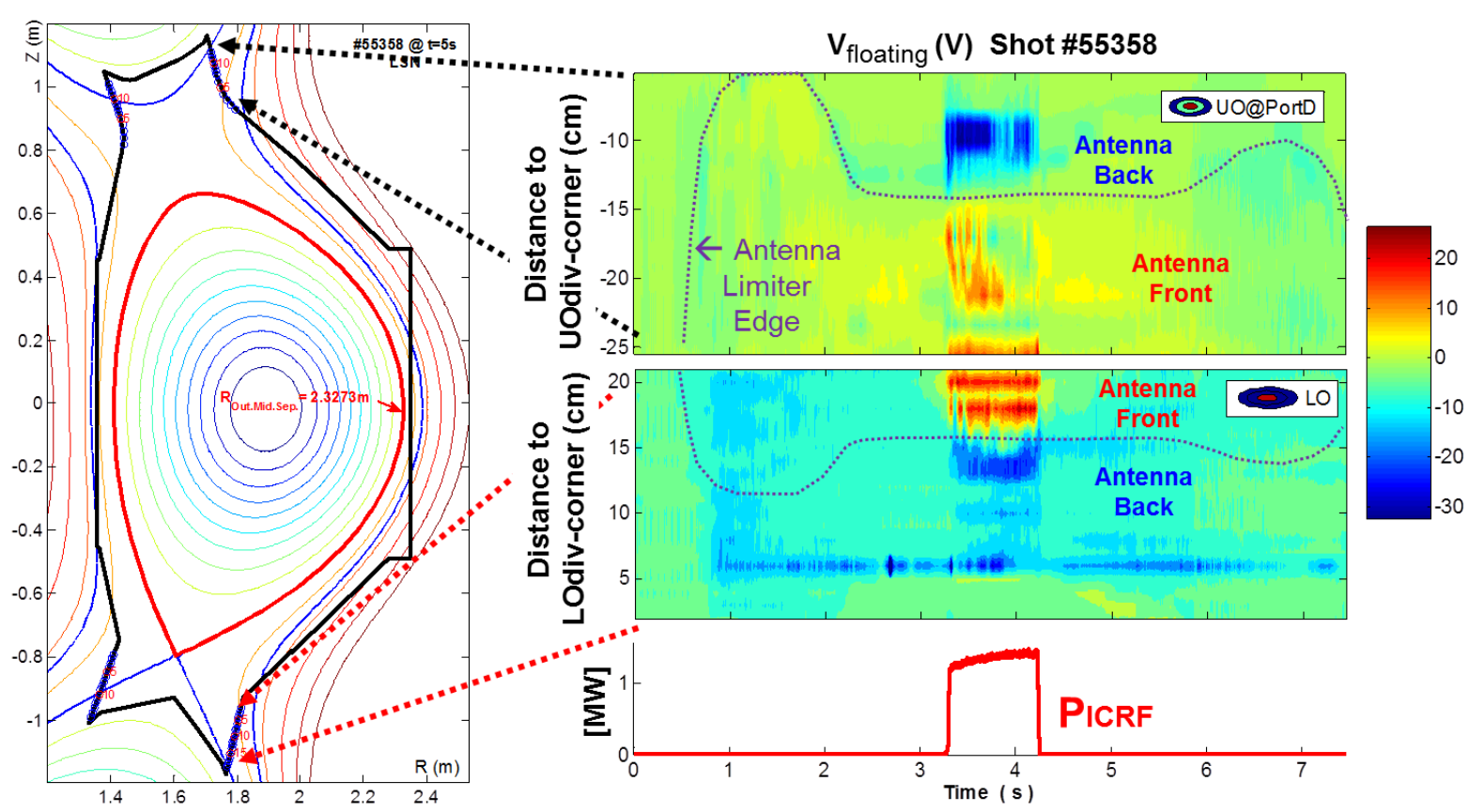

Figure 11. Time evolution of Langmuir probes floating potential $V_{f}$ profiles of both upper and lower divertors outer targets during an LSN discharge. Regions magnetically connected to the front and the back of the antenna limiter edge are separated by the dotted purple curve.

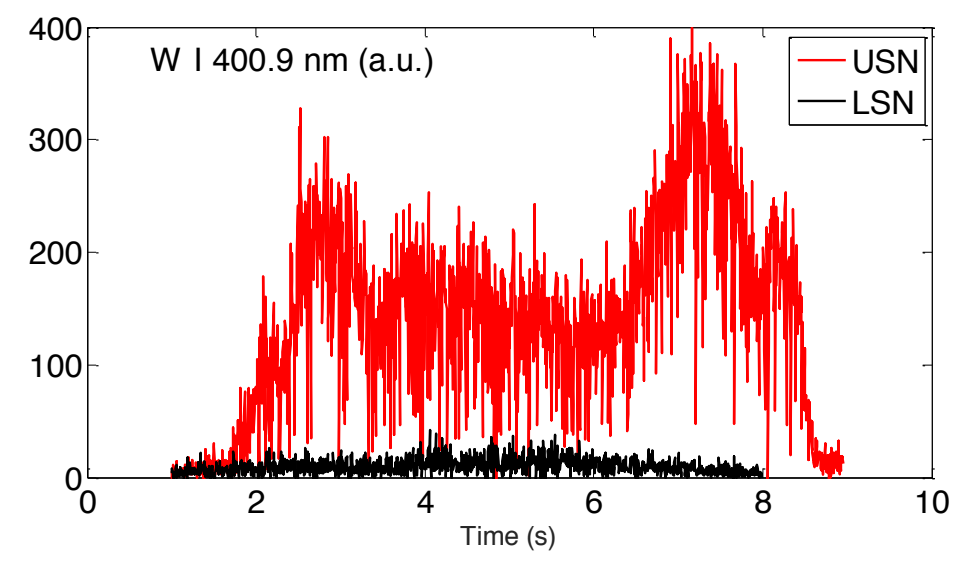

Figure 12. Time evolution of WI emission (at 400.9nm) measured by visible spectrometer at upper outer divertor target in both LSN (\#55358) and USN (\#69671) magnetic configurations

\subsection{Near-field effects on main chamber objects magnetically connected to active antennas}

Surfaces close and magnetically connected to active ICRH antennas are often subject to much stronger interactions with plasma due to RF sheath excitation [42]. Fig.13 represents $\mathrm{Ti}$ (magnetically connected to B-port) and Fe (present in antennas Faraday screens) as functions of both PICRH and $\mathrm{P}_{\text {TOT }}$ such as in Fig.8.

As expected from Fig.3a and Fig.3b, magnetically connected species are well correlated to

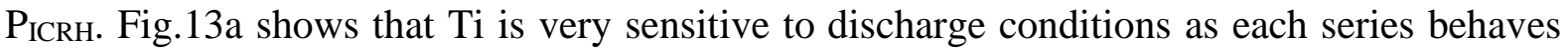
differently, whereas Fe has different behaviours but globally increases with PICRH (Fig.13b). At constant LH power and same ICRH power on each strap (blue series), Ti and Fe both follow

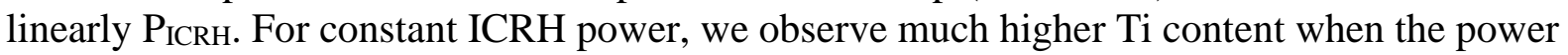
is unbalanced (red series) than when it is well balanced (blue series). When ICRH increases together with LH power (black series), Ti remains constant, demonstrating that interactions in regions closely connected to the B-port antenna may not only depend on ICRH power but also on how it is launched (antenna design, power balance on straps). Fe from antenna Faraday 
screens always increases with ICRH power in a similar fashion in all cases showing a generally good correlation. As shown by the red series while changing the power balance of the B-port antenna, Fe sensitivity to one single antenna's settings (Fig.13b) is however less obvious than for Ti (Fig.13a), since Fe can come from both antenna Faraday screens.

In Fig.13c and Fig.13d representing $\mathrm{Ti}$ and Fe increase as function of $\mathrm{P}_{\mathrm{TOT}}$, the red series - representing discharges with unbalanced power on the straps - is significantly above the others, whereas the power balance does not clearly affect the divertor region as shown in Fig.8. For magnetically connected species, in the absence of ICRH, the impurity concentration seems independent of the $\mathrm{LH}$ power, at least in the 1-2.5MW power range. This confirms $\mathrm{Ti}$ and Fe closely connected to antennas are mostly sensitive to ICRH settings and near-field effects.
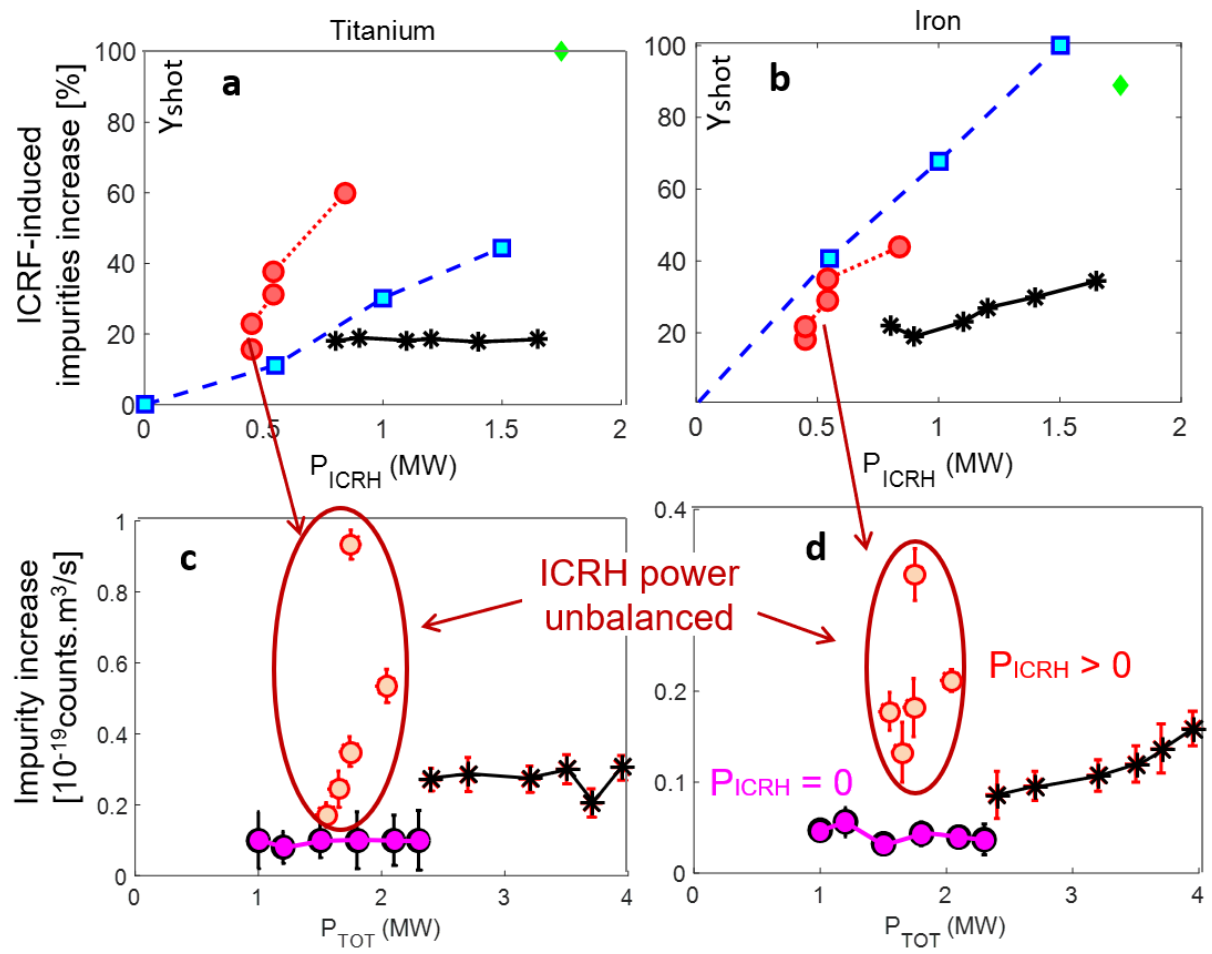

Figure 13. (a and b) Ti and Fe production ( $Y_{\text {shot }}$ as defined in eq.1) as a function of ICRH power and (c and d) $\mathrm{Ti}$ and $\mathrm{Fe}$ increase normalized by electron density against total power.

Blue, red and black series in graphs a and b correspond to the same discharges as in Fig.7. Graphs $\mathrm{c}$ and d also represent the same discharges except ones of the blue series, in addition points without ICRH (magenta series) were added (adapted from [12]).

Two other stainless-steel plates (coated with a $5 \mu \mathrm{m}$ layer of Ag (silver)) were fixed in 2018 on one limiter of the I-port antenna (the one on the H-port side, cf. Fig.14). This region was chosen given that it is very well connected to the I-port and pretty much nothing else. The limiter between $\mathrm{G}$ and $\mathrm{H}$ ports being radially $1 \mathrm{~cm}$ closer to the plasma than the antenna limiters, no fast electron accelerated by the LH launcher in E-port can either reach this region. However, the plates melted at each corner over about $4 \mathrm{~cm}$ poloidally, precisely where HFSS model predicts peaks of RF potential due to sheath excitation [23]. Furthermore, as shown in Fig.14, the part that melted on each plate (represented by the stars) is distinct from the one that was the closest to the plasma (represented by the circles). This is therefore an example of the consequences near-fields excited RF sheath may have on plasma surface interactions.

Knowing all the characteristics of the plate and that stainless steel would start melting between 1300 and $1400^{\circ} \mathrm{C}$, a thermal analysis made with ANSYS - which details are given in Appendix - provides an estimation of the minimal heat flux that may have induced the plates to melt. Assuming the heat fluxes were mostly parallel, a parallel flux above $5 \mathrm{MW} / \mathrm{m}^{2}$ would suffice to induce partial melting of the plate in less than a second. Identifying \#78578 as one of the most likely discharge of 2018 campaign during which such conditions might have been 
reached (1.7MW on I-port with $60^{\circ}$ phasing and power unbalanced on the straps), we can estimate SOL electron temperature $\left(\mathrm{T}_{\mathrm{e}} \approx 20 \mathrm{eV}\right)$ and density $\left(\mathrm{n}_{\mathrm{e}} \approx 3.10^{17} \mathrm{~m}^{-3}\right)$ with nearby Langmuir probes. Following the simplest models in available literature [44] and assuming that parallel heat fluxes are entirely due to DC potential drop across the sheath [45], it is finally possible to estimate the DC potentials that would have been necessary to reach such heat fluxes through the parametric scaling

$$
V_{D C \text { sheath }}=\frac{Q_{\|}}{\Gamma_{\text {Bohm }} e} \approx \frac{Q_{\|}}{n_{e} \sqrt{T_{e}+T_{i}}} \approx \frac{Q_{\|}}{n_{e} \sqrt{3 T_{e}}}=600 \mathrm{~V}
$$

$600 \mathrm{~V}$ happens to be a value pretty consistent with typical potentials measured by Langmuir probes (several hundred volts) not specifically connected to the region of the maximum.
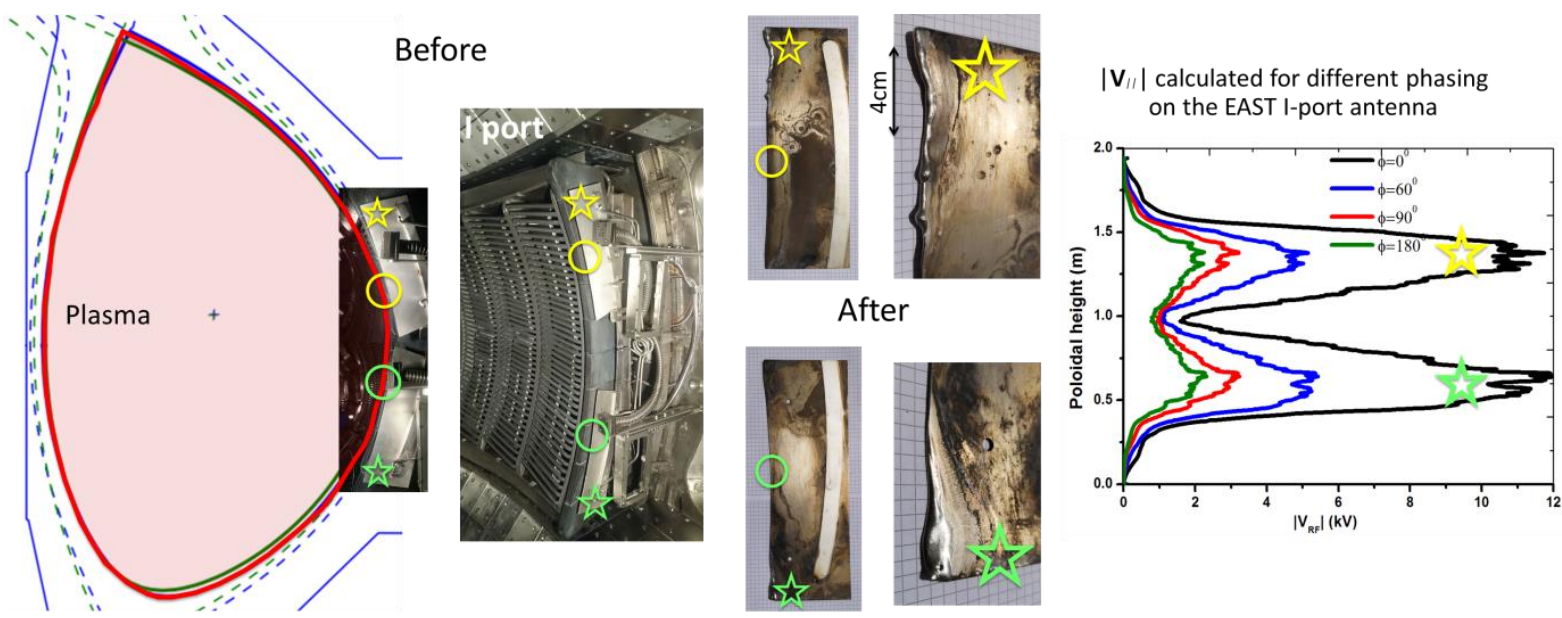

Figure 14. Pictures of the plates fixed on I-port antenna limiter, before and after 2018 campaign. Green and yellow circles represent respectively the part of the lower and upper plate that is the closest radially, while the stars represent the region that melted. The graph to the right shows that maximum of sheath potential computed with HFSS are precisely expected in the region where plates melted. On pictures taken after the experiment, plates are superposed on a grill of $5 \mathrm{~mm}$ squares to give an idea of the dimensions.

In order to better represent the impurity correlation with $\mathrm{P}_{\mathrm{ICRH}}$, we focus on the black series based on maximum number of points and for which each species shows significantly different behaviours. For those discharges, ICRH-related impurity production is now represented relatively to the species with the highest ratio $\mathrm{P}$ - representative of how likely a given species is to contaminate core plasma when turning ICRH on - which happens to be Fe amongst Ti and W (Fig.15).

$$
P=\left(\operatorname{Imp} p_{\text {withIC }}-\operatorname{Im} p_{\text {without IC }}\right) / \mathrm{m} \text { ean }\left(\operatorname{Imp} p_{\text {withIC }}-\operatorname{Im} p_{\text {without IC }}\right)_{\text {overblack shots }}
$$

The indicator of correlation is now noted $Y_{\text {species }}$ and is calculated as

$$
Y_{\text {species }}=\left(I m p_{\text {with IC }}-\operatorname{Im} p_{\text {without IC }}\right) / \max (\mathrm{P})_{\text {over all speciesand shots }}
$$

Error bars correspond to the root mean square of $Y_{\text {species }}$ for the time periods during which $\operatorname{Imp}_{s}$ are assessed. This quantity is basically representative of each species' correlation with PICRH. Fig. 15 typically shows that Fe is the species that best correlates with PICRH which is consistent with the fact that it mostly comes from antenna Faraday screens where the strongest near-fields are expected. Ti farther away from antennas and magnetically connected to the B-port increases in presence of ICRH, independently on its power, but mechanisms other than just RF sheath excitation seem to play a role, as simply increasing $\mathrm{P}_{\text {ICRH }}$ does not necessarily acts on Ti production. $\mathrm{W}$, an element that used to be found only in components in the divertor region, appeared weakly correlated to ICRH power compared to Fe. But this situation changed in 2018 when both LH launchers guard limiters were changed into tungsten 
(Fig.1). Comparing both situations ultimately allows us to quantify these limiters contribution to core $\mathrm{W}$ content.

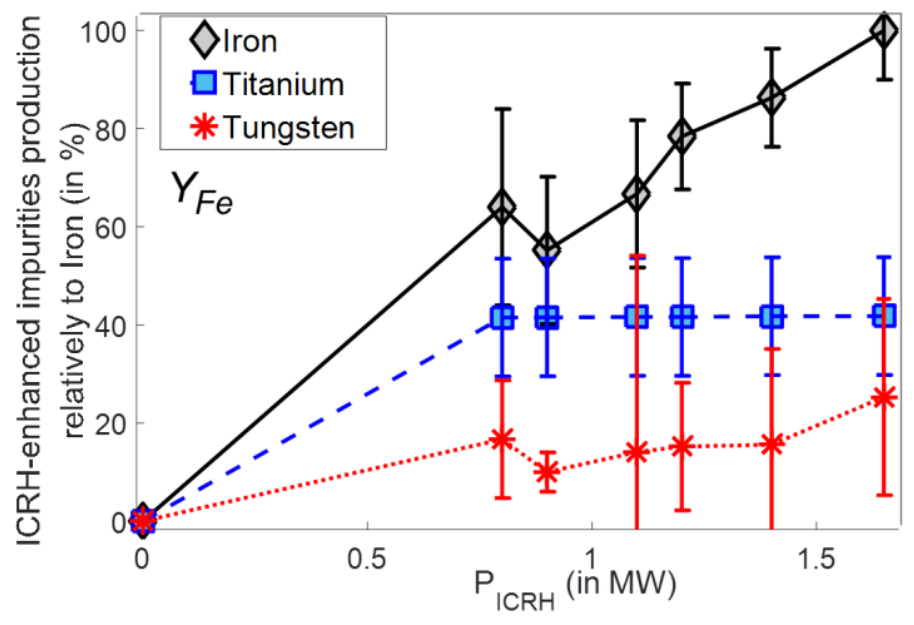

Figure 15. ICRH impurities production increase relatively to $\mathrm{Fe}$ $\left(Y_{F e}\right)$ as a function of ICRH power

\section{Quantify the contribution of $\mathrm{LH}$ grill limiters to core $W$ contamination in EAST from LSN/USN comparisons.}

Up to this point, it has not been possible to quantify the contribution of different elements of the chamber due to the core contamination by tungsten. In 2018, one of the main upgrades made in EAST concerned both LH launchers' lateral protections which used to be composed of graphite tiles and were fully replaced by tungsten tiles with different designs on each grill (Fig.16b). Like in other devices like ASDEX Upgrade [46], having W at the mid-plane substantially changes the impurity production picture we got in the previous sections, and this will now be compared to complement the discussion before concluding. As shown in Fig.12 by visible spectroscopy data providing a rather direct estimation of $\mathrm{W}$ emission at the divertor, much stronger erosion occurs in USN rather than LSN. This is consistent with core W content whose ratio in USN against LSN are plotted in Fig.16a. The black dotted curve represents the core W content ratio between similar discharges in USN versus LSN when W was still characteristic of divertor region, typically showing that erosion rate in USN was about 8 times higher than in LSN. The red solid curve represents the same quantity after the 2018 upgrade for discharges without ICRH (\#77724 over \#77722). Having W at the mid-plane led to homogenization of $\mathrm{W}$ core contents in different configurations, suggesting that the contamination efficiency by divertor sources is negligible compared to the mid-plane one. 

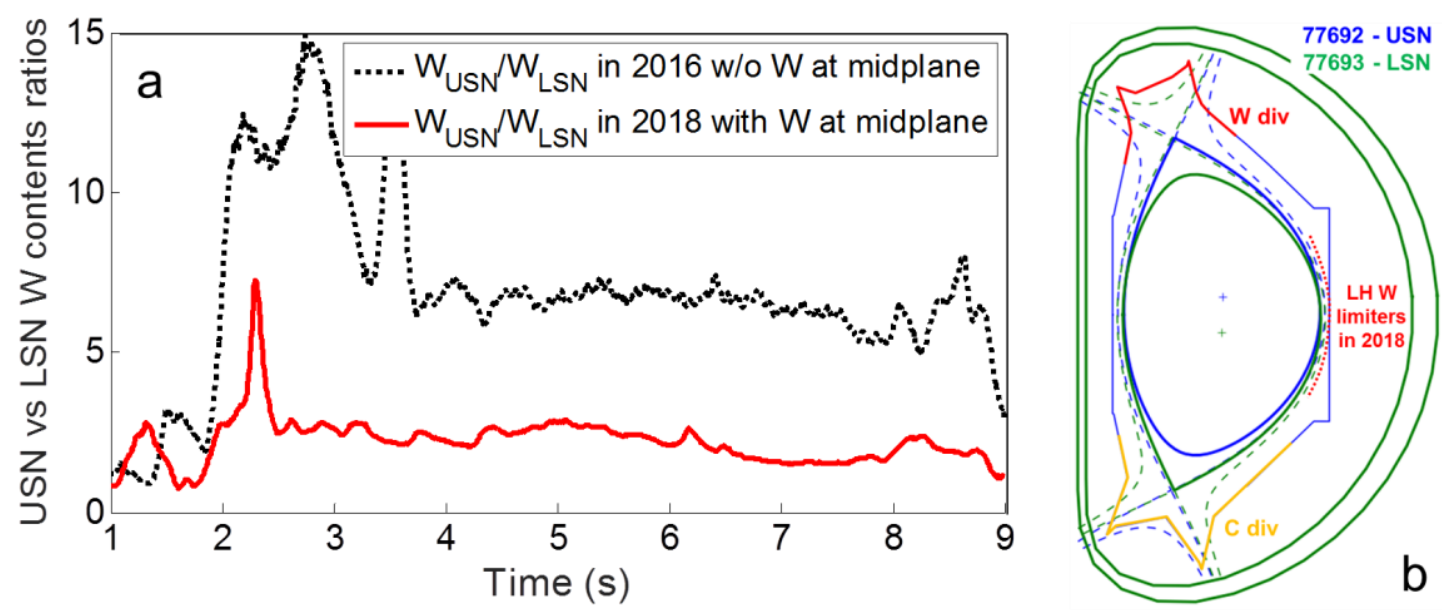

Figure 16. (a) Comparison of $W$ contents ratios between similar discharges without ICRH in USN and LSN configurations, with (2018 red solid curve) and without (2016 black dashed curve) W present at midplane. (b) USN vs LSN EFIT magnetic equilibriums

In order to quantify the contribution of LH limiters to core contamination by $\mathrm{W}$, we will make the following assumptions:

- A0) Core temperature are similar in LSN and USN such that the ratio of brightness in LSN and USN are representative of W concentration.

- A1) The core W content on EAST can be decomposed into 3 contributions:

- W1 from W upper divertor

- W2 from LH limiters

- W3 from "elsewhere" in the main chamber (unknown...)

With these notations, we basically want to estimate the relative contribution of $\mathrm{LH}$ limiters to the core contamination, i.e. the ratio $\mathrm{W} 2 /(\mathrm{W} 1+\mathrm{W} 2+\mathrm{W} 3)$.

- A2) W2=0 before the tungstenisation of LH limiters.

- A3) W2 and W3 remain the same in USN and LSN configurations.

- A4) W1=0 in LSN configuration.

- A5) W3/W1 is the same before and after tungstenisation of LH limiters.

Assumptions A3 and A4 are used to discriminate the location of the sources. They imply that there is no W source (e.g. specific from the lower divertor) that would appear only in LSN configuration. A2 is necessary to discriminate LH limiter contribution from the rest. A5 means that $\mathrm{LH}$ limiters tungstenization is the main change in the machine, ideally the only one.

Under the above assumptions, USN vs LSN comparison before tungstenization of the LH limiters estimates $\mathrm{W} 3 / \mathrm{W} 1$ :

$$
\left(\frac{L S N}{U S N}\right)_{\text {before }}=\frac{1}{1+W 1 / W 3}
$$

USN vs LSN comparison after tungstenization estimates (W3+W2)/W1.

$$
\left(\frac{L S N}{U S N}\right)_{\text {after }}=\frac{1}{1+W 1 /(W 2+W 3)}
$$

Whence the estimated ratio $\mathrm{W} 2 /(\mathrm{W} 1+\mathrm{W} 2+\mathrm{W} 3)$ without ICRH leads to

$$
\frac{W 2}{W 1+W 2+W 3}=\frac{\left(\frac{L S N}{U S N}\right)_{\text {after }}-\left(\frac{L S N}{U S N}\right)_{\text {before }}}{1-\left(\frac{L S N}{U S N}\right)_{\text {before }}}=\frac{\frac{1}{3}-\frac{1}{8}}{1-\frac{1}{8}} \approx 20 \%
$$


Note values above 70\% were observed in presence of ICRH in ASDEX Upgrade [47]. Unfortunately, this could not be assessed in EAST because the lower divertor was not used at all during most recent experimental campaigns.

Note the actual ratio probably depends on the level of ICRH power compared to the total additional power, on the fraction of ICRH power emitted by each ICRF antenna, on the strap phasing, Li conditioning ...

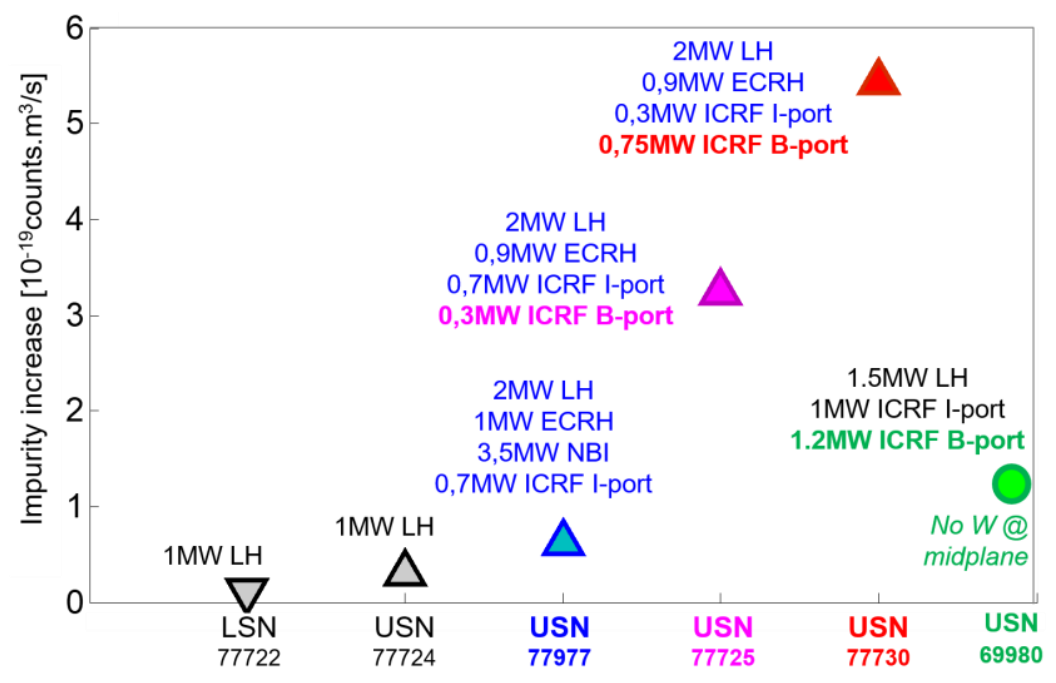

Figure 17. With W present at mid-plane, comparison of W contents in one LSN and four USN discharges with different heating scenarios. The large difference when B-port ICRH antenna is powered suggests that main sources are magnetically connected LH launchers limiters.

W content in the core for the two same discharges heated with 1MW LH power during the 2018 campaign were represented by black triangles in Fig. 17. The small difference between these similar discharges in different configuration $\left(\mathrm{W}_{\mathrm{USN}}<2 . \mathrm{W}_{\mathrm{LSN}}\right.$ ) suggests principal $\mathrm{W}$ sources are now located at mid-plane. Other USN discharges with different heating scenarios were displayed on the same graph. The blue triangle stands for a discharge heated with all heating systems except the B-port ICRH antenna and we see, despite the relatively high amount of power injected (7.2MW in total with about half from NBI), that the W level remained relatively low. In another discharge however (magenta triangle), the $3.5 \mathrm{MW}$ from NBI were replaced by $0.3 \mathrm{MW}$ ICRH from B-port, and the W content rose more than fivefold. Finally, another discharge followed with similar total power injected but having $350 \mathrm{~kW}$ ICRH injected from the B-port rather than from the I-port (red triangle), which led to another sharp increase of the $\mathrm{W}$ content. These results are consistent with magnetic connections existing between ICRH antennas and LH launchers limiters. As can be seen on Fig.1, the I-port antenna only connects to the upper part of the N-port LH grill and does not connect to the E-port grill shadowed by the limiter between the $\mathrm{G}$ and $\mathrm{H}$ ports. The B-port antenna is however wellconnected to both LH launchers, therefore enhancing interactions with $\mathrm{W}$ limiters. These results together show that $\mathrm{W}$ sources in a mid-plane region magnetically connected to an active ICRH antenna can lead to severe core contamination by W. To put it differently, this experiment proves that the ICRH antennas should have no magnetic connection with a normal incidence to objects inside the vacuum chamber.

\section{Discussions and prospects}


By using the EUV spectrometer to characterize the presence of high- $\mathrm{Z}$ metallic impurities in the core, and benefiting from the multiplicity of materials in EAST, we were able to deduce local information about ICRH-related plasma surface interactions.

Magnetic connection between objects of specific composition and active antennas undeniably plays a role which becomes more and more complex as the distance to active antennas increases. Fe from antenna Faraday Screens typically correlates well with ICRH power, which is believed to be the result of near-field enhanced interactions, so far consistent with most observations elsewhere [3-13]. Rectification effects are then transported along magnetic field lines from active antenna to connected regions, as observed through the $\mathrm{Ti}$ content evolution when degrading the power balance on antenna straps. This observation is consistent with uncompensated image currents induced on antenna limiters, leading to stronger potential rectification at the Ti plates. Besides, for well-balanced power on straps, ICRHinduced rectification can be inhibited in the same region. This could additionally be the result of improved wave absorption by increasing total injected power which would require a dedicated study including more precise calculations of wave absorption efficiency. When W was still exclusive to the upper divertor region, it used to show much better correlation with total conducted power rather than with ICRH power, or settings like power balance or phasing between straps. Divertor outer target was identified in others devices to be an important source of impurities [15, 16, 32] but not specifically due to ICRH [14, 17]. In EAST, potential rectifications are observed by divertor Langmuir probes. These increase together with ICRH power and occur in comparable fashion either in LSN or USN. On the contrary, erosion rates are very different and depend on magnetic configuration, which leads to the belief that, unlike observation from other experiments $[15,16,38]$, the poor level of ICRH power injected in EAST may not suffice in the divertor region to rectify the sheath up to the critical regime of sputtering (Fig. 1 in [16]). At the mid-plane, however, the connection length to the ICRH antennas and the SOL width being much shorter than in the divertor region, both RF-induced erosion rate [17] and impurities penetration [15] are higher. Therefore, changing lateral protections of the two LH launchers from graphite into $\mathrm{W}$, leads to comparable core contamination by $\mathrm{W}$ in different magnetic configurations, meaning dominant impurity sources are located at mid-plane. Moreover, mid-plane erosion of $\mathrm{W}$ is particularly strong when connected ICRH antenna is powered. As far as EAST L-mode plasmas are concerned, we therefore conclude that RF sheath is the mechanism that widely dominates plasma materials interactions and core contamination by impurities. We even collected evidence of metallic plates melting induced by RF sheath excitation on antenna corners, which also motivates further study based on camera images to extract information on how impurity penetrate inside the plasma.

Most metallic impurities were moreover insensitive to ICRH phasing that was not feedback controlled [25], which is not consistent with similar experiments and modeling predictions $[1,11,32]$ as shown in appendix 2. Only Mo reacted to phase changes with increasing interactions over phase scan from dipole to monopole. If Mo were to come from low field side due to near fields, Fe should have similar behavior, which was not the case. We therefore believe Mo comes from the part of the high field wall facing I-port antenna. This hypothesis is consistent with wave propagation and absorption modeling with EVE [35] and TOMCAT codes [34]. As phasing decreases from dipole to monopole; (i) single pass absorption efficiency shrinks from $55 \%$ down to $15 \%$, (ii) low $\mathrm{k}_{\|}$modes are excited for phasing bellow $60^{\circ}\left(\sim \mathrm{k}_{\|}<3 \mathrm{~m}^{-1}\right)$, and (iii) electric field amplitude increase at the high field side, likely enhancing plasma surface interactions on Mo target.

It should be pointed out that the two ICRH antennas have different designs and sizes, meaning that not only the magnetic connection but also the antenna design can both play a role in impurity generation [9]. Note that the B-port is systematically the principal cause of impurity production up to deleterious levels, which can be partially understood considering its smaller 
size compared to the I-port, leading to higher power density for similar generator power and loading.

Note that impurity transport was considered fairly constant in the L-mode discharges discussed in the present study, allowing to interpret impurity changes in the core as changes in the source intensity. The next step will be to extend this study to H-mode discharges, but the approach will have to be different as impurity transport can then be subject to strong changes. A new spectrometer should typically be used for measuring radial profiles of various impurity in $\mathrm{H}$-mode [51].

\section{Appendix}

\section{A1) Complements on EVE and TOMCAT modelling of ICRH wave propagation and absorption in EAST}

TOMCAT 1D [34] was used to quickly estimate wave absorption. It guarantees that a positive definite power transfer from waves to particles ensured for any of the wave modes in plasma with all species following analytical Maxwellian distributions, as is expected from first principles. Rather than relying on a truncated Taylor series expansion of the dielectric response, an integrodifferential approach with finite Larmor radius assumption of the Kennel-Engelmann operator up allows retaining effects up to $\mathrm{N}=3$ harmonic. relies on heating rather than of the dielectric tensor To keep the required computation time for this generalized description reasonable, tabulation of integrals is intensively used.

EVE 3D [35] was used to get a detailed view of how wave propagates in the plasma. Local RF electric field structure are obtained by the full-wave computations consisting in calculating the wave field by a direct solution of the Maxwell's equations in the Fokker-Planck description with variational formulation described in [35]. It is based on the quasilocal plasma functional obtained in terms of adiabatic invariants, in which an expansion of the particle Hamiltonian is performed to yield a tractable second-order finite Larmor radius version of this functional that was implemented. Simplistic antenna straps geometry can then be prescribed with key parameters settings (ex: phasing) from which a spectrum discretized in harmonics for each toroidal number is deduced before being loaded with given target plasma defined by its composition $\left(\mathrm{Z}_{\text {eff }}=1.8\right)$, aspect ratio $\left(\mathrm{a}=0.44 \mathrm{~m}, \mathrm{R}_{0}=1.85 \mathrm{~m}\right)$, magnetic equilibrium, electron temperature and density profiles $\left(\mathrm{T}_{\mathrm{e}}(\mathrm{r})\right.$ and $\left.\mathrm{n}_{\mathrm{e}}(\mathrm{r})\right)$ for reference discharge \#69950.

In order to better enlighten wave properties in EAST, Fig.A1 represents fields polarization in different regions and for different phasing computed by EVE [35] as phasing decreases down to monopole.
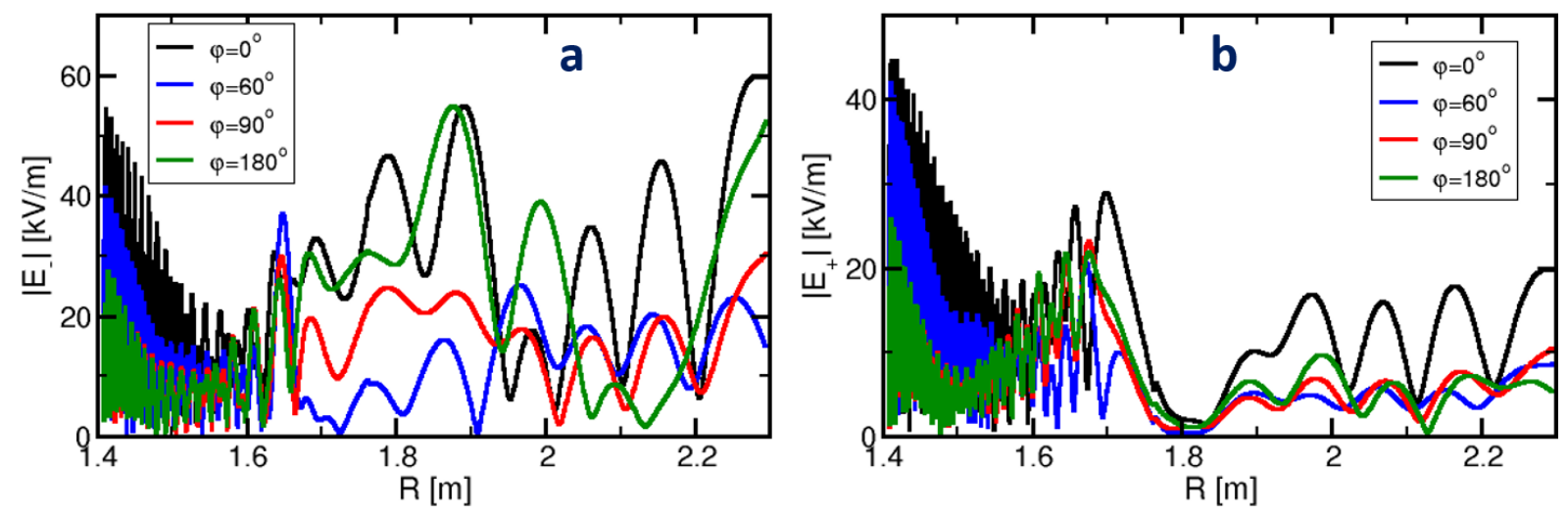

Figure A1. Radial evolution of the amplitudes of the (a) negative and (b) positive polarizations of the electric field (in V/m) computed with EVE code, adapted from 
Fig.A2 also shows $\boldsymbol{k}_{\|}$spectra in vacuum and loaded by EAST plasma. It can be clearly seen on the loaded spectrum that a low $\boldsymbol{k}_{\|}$mode is excited bellow $60^{\circ}$ phasing. Low $\boldsymbol{k}_{\|}$mode absorption might be poor, potentially leading to proper modes in the plasma and power dissipation into the SOL with deleterious impact on plasma surface interaction.
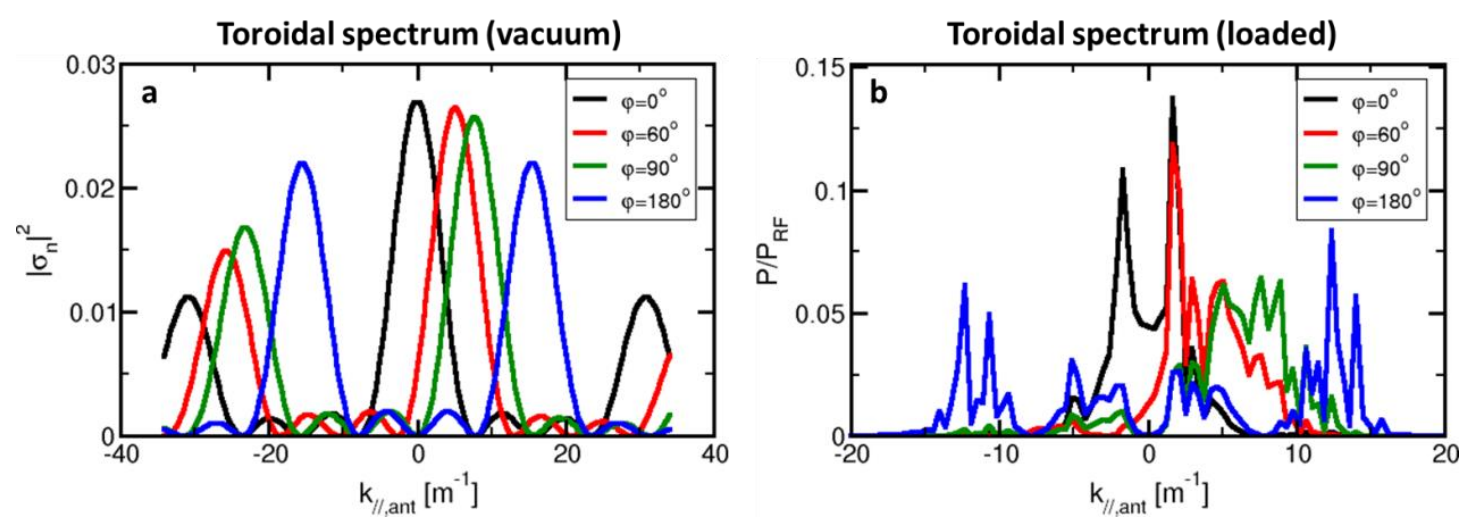

Figure A2. Antenna toroidal spectrums for different strap phasing $\left(0^{\circ}, 6^{\circ}, 90^{\circ}, \mathbf{1 8 0}^{\circ}\right)$,

(a) in vacuum and (b) in front of EAST plasma load (reference discharge 69950) computed with EVE.

\section{A2) ANSYS estimation of RF-sheath induced heat fluxes on I-port antenna corners necessary to induce metallic plates melting}

In this simulation with ANSYS, we consider an inertial stainless-steel plate of $30 \mathrm{~cm}$ (poloidally) x $10 \mathrm{~cm}$ (radially) x $1 \mathrm{~mm}$ (toroidally). The heat flux is assumed parallel (toroidal) and its maximum is applied as observed after the experiment, $3 \mathrm{~cm}$ away from the corner of the plate (Fig. A3). From this maximum, heat fluxes decrease exponentially in both radial and poloidal directions, as shown in Fig.A3b per focusing mostly on RF sheath induced heat flux.

Dynamic simulations where made for five different values of maximal heat flux from 1 to $10 \mathrm{MW} / \mathrm{m}^{2}$, and plate maximal temperature evolution represented in Fig.A4. Note values estimated in Tore Supra and JET would typically be of the order of 2 to $7 \mathrm{MW} / \mathrm{m}^{2}$ [45]. In EAST, these simulations predict that plate would have started melting after about 30s for a maximal heat flux of $1 \mathrm{MW} / \mathrm{m}^{2}$, after $2.3 \mathrm{~s}$ for $3 \mathrm{MW} / \mathrm{m}^{2}$, and in between 1 and $2 \mathrm{~s}$ (typical length of ICRF pulses in EAST) for maximal heat flux of $5 \mathrm{MW} / \mathrm{m}^{2}$.

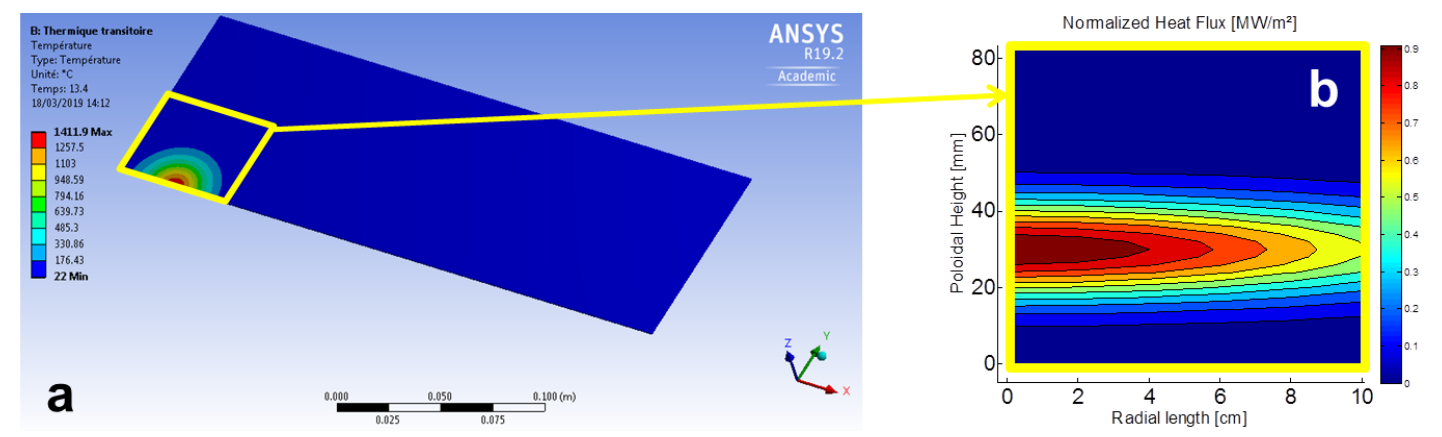

Figure. A3. (a) ANSYS model with (b) heat loads pattern on the metallic plate corner 


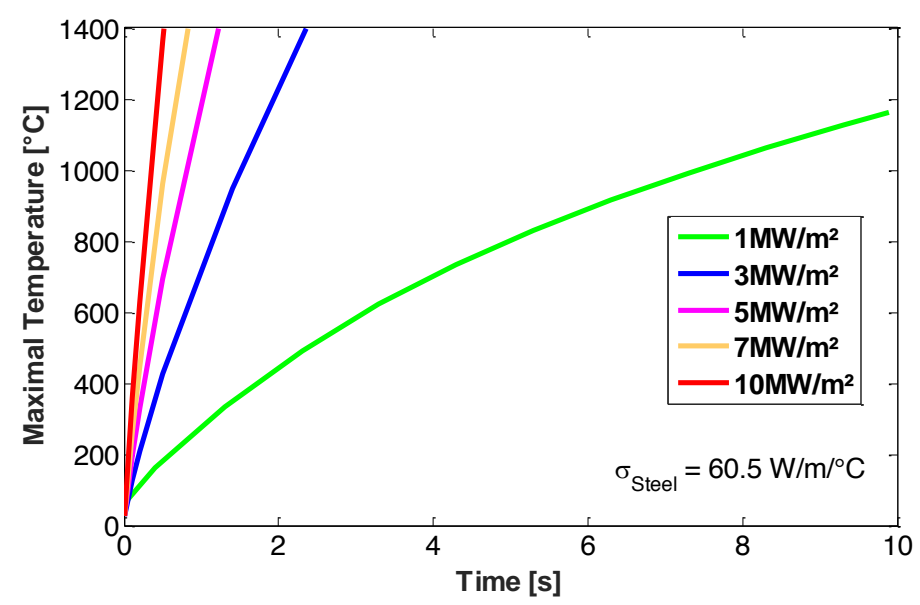

Figure A4. Evolution of the maximal temperature of the plate for different parallel heat fluxes calculated with ANSYS. Steel plate starts melting when its temperature approaches $1400^{\circ} \mathrm{C}$.

\section{A3) Comparison of surface temperature estimations from infrared and temperature inversion with TEDDY code on EAST I-port graphite limiter tile}

Despite no infrared camera was looking at the metallic plates shown in Fig.14, a wide-angle camera $\left(\mathrm{FoV}=62^{\circ} \times 59.5^{\circ}\right)$ is fixed on K-port and its angle was changed in 2019, allowing good resolution infrared measurements on the I-port antenna limiter (Fig.A5) with $640 \times 512$ pixels full frame at $115 \mathrm{~Hz}$ with pixel size on antenna limiter 3-4mm [48]. During discharge 83746 made in 2019, the power was far from balanced on the straps with power changing between $50 \mathrm{~kW}$ up to $350 \mathrm{~kW}$ from one strap to its neighbour. Already suspecting from past year that the plate most likely melted due to this type of setting unfavourable for RF sheath excitation, we analysed the apparent temperature on the antenna bottom corner symmetric to where the plate melted. The temperature remained fairly low along the discharge and saturated at about $300^{\circ} \mathrm{C}$ in about $20 \mathrm{~ms}$ as the antenna was powered. Visible on the video, spectacular graphite dusts were also emitted from the hot spot with surprisingly deep penetration before getting ionized and starting following magnetic flux tubes. This peculiar observation is not yet understood and will be the object of future investigation. In order to get an estimation of which heat flux would have been necessary to induce such apparent temperature increase on the infrared camera, we used TEDDY code [49] recently developed in CEA. TEDDY is 2D thermal model inspired of THEODOR [50] that can calculate a surface temperature distribution for a given heat flux distribution in the direct method. The apparent temperature - IR measurement $(\lambda=3-5 \mu \mathrm{m})-$ is calculated from surface temperature using emissivity $(\varepsilon=0.7)$ and reflection of the ambiance corrections $\left(\mathrm{T}_{\mathrm{amb}}=25^{\circ} \mathrm{C}\right)$. By prescribing a $10 \mathrm{MW} / \mathrm{m}^{2}$ heat flux on the target with similar pattern as the one shown in Fig.A5b. we see that we recover the same apparent temperature increase as the one observed by the IR camera. Sheath effect induce steep temperature profiles regarding the pixel size, which induces a smoothing effect on the temperature not taken into account in TEDDY. The maximum apparent temperature shown with the IR is lower due to smoothing effect which means that the heat flux required should be higher than the $10 \mathrm{MW} / \mathrm{m}^{2}$ mentioned above. Note finally this heat flux is twice larger than the one that would have been necessary to melt the plates based on ANSYS estimations. 

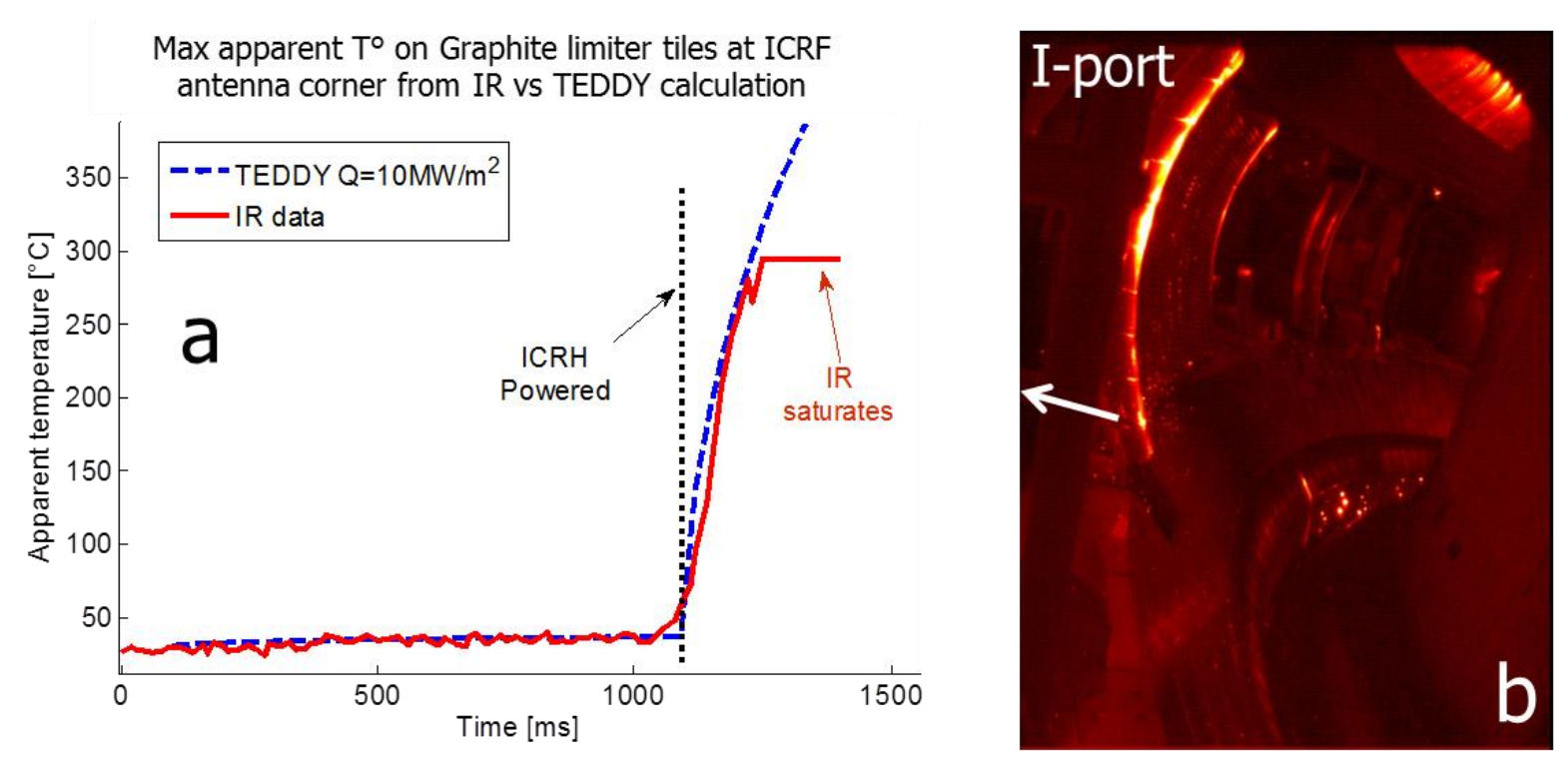

Figure. A5. EAST I-port ICRF graphite limiter corner maximal apparent temperature estimation from infrared camera (red solid curve) superposed onto temperature increase induced by $10 \mathrm{MW} / \mathrm{m}^{2}$ heat fluxes and estimated with TEDDY code (blue dotted curve).

\section{Acknowledgments}

The whole EAST ICRH team, colleagues in charge of reciprocating probes and IRFM GMICS team are warmly acknowledged.

This work was supported partly by National key research and development program (grant No.2016YFA0400600, 2016YFA0400601 and 2018YFE0311100). This work was also supported partly by the National Natural Science Foundation of China under grant nos 11675213,11975265 and 11775269.

This work has been carried out within the framework of the the French Federation for Magnetic Fusion Studies (FR-FCM) and EUROfusion Consortium and has received funding from the Euratom research and training program 2014-2018 and 2019-2020 under grant agreement No 633053. The views and opinions expressed herein do not necessarily reflect those of the European Commission.

This work was carried out under the framework of the Sino-French Fusion Energy centre (SIFFER, http://www.siffer.science/).

\section{References}

[1] J.H. Zhang et al. 2017 Nucl. Fusion 57066030

[2] Y. Q. Yang et al. 2017 Plasma Phys. Control. Fusion 59095001

[3] G. Urbanczyk et al. EPJ Web of Conferences 157, 03057 (2017)

[4] L. Colas et al. 2007 Plasma Phys. Control. Fusion 49 B35

[5] M. Kubic et al. Journal of Nuclear Materials 438 (2013) S509-S512

[6] R. Ochoukov et al. 2014 Plasma Phys. Control. Fusion 56015004

[7] I. Cziegler et al. 2012 Plasma Phys. Control. Fusion 54105019 
[8] R. Hong et al. 2017 Plasma Phys. Control. Fusion 59105008

[9] V. Bobkov et al. Journal of Nuclear Materials 438 (2013) S160-S165

[10] V. Bobkov et al. 2017 Plasma Phys. Control. Fusion 59014022

[11] M.J. Martin et al. PRL 119, 205002 (2017)

[12] G. Urbanczyk et al, Nuclear Materials and Energy 17 (2018) 274-278

[13] V. Bobkov et al. Nuclear Materials and Energy 18 (2019) 131-140

[14] R. Dux Journal of Nuclear Materials 390-391 (2009) 858

[15] B. Lipschultz et al. Nucl. Fusion 41, p.585 2001

[16] S. Wukitch et al. Journal of Nuclear Materials 390-391 (2009) 951-954

[17] R. Dux et al. Journal of Nuclear Materials 363-365 (2007) 112-116

[18] L. Zhang et al. 2015, Review of Scientific Instruments, 86, 123509

[19] Z. Xu et al. 2018 Nucl. Fusion 58016001

[20] K. Y. Chen et al. Rev. Sci. Instrum. 87 (2016) 063504

[21] H. Mao et al. Nuclear Materials and Energy 12 (2017) 447-452

[22] M. H. Li et al. Physics of Plasmas 23, 102512 (2016)

[23] Qin Chengming et al. Plasma Science and Technology, Vol.17, No.2, Feb. 2015

[24] J.H. Wang et al. Fusion Engineering and Design 122 (2017) 196-203201

[25] Z. Chen et al. Nuclear Science and Technology (2018) 2919

[26] N Yan et al. 2013 Plasma Phys. Control. Fusion 55115007

[27] R.S. Granetz et al. Journal of Nuclear Materials 241-243 (1997) 788-792

[28] G.Y. Antar et al. 2012 Nucl. Fusion 52103005

[29] S. Wukitch et al APS Division of Plasma Physics Meeting 2014 DPPUO3012W

[30] V. Bobkov et al, Nuclear Materials and Energy 12 (2017) 1194-1198

[31] M. Valisa et al. 2011 Nucl. Fusion 51033002

[32] K. K. Kirov et al. 2009 Plasma Phys. Control. Fusion 5104400

[33] E. Lerche et al. AIP Conference Proceedings 1187, 93 (2009)

[34] D. Van Eester and E. Lerche. Plasma Phys. Control. Fusion 55 (2013) 055008

[35] R. Dumont et al. 2009 Nucl. Fusion 49075033

[36] G. Urbanczyk et al. AIP Conference Proceedings AIPCP20-AR-RFPPC2019-00013 "ICRH coupling optimization and impurity behavior in EAST and WEST"

[37] S. Shiraiwa et al. 2017 Nucl. Fusion 57086048

[38] R. Perkins et al. Physical Review Letters 109(4) July 2012

[39] M.Vrancken et al. Fusion Engineering and Design 88 (2013) 940-944

[40] J.C. Xu et al. 2016, Review of Scientific Instruments 87, 083504

[41] R. Perkins et al. EPJ Web of Conferences 157, 03039 (2017)

[42] R. Perkins et al. 2019 Plasma Phys. Control. Fusion 61045011

[43] E A Lerche et al 2008 Plasma Phys. Control. Fusion 50035003

[44] F.W. Perkins 1989 Nucl. Fusion 29583

[45] L Colas et al. AIP Conference Proceedings (2009) Vol. 1187 Issue 1, p133

[46] V. Bobkov et al. 2010 Nucl. Fusion 50035004

[47] V. Bobkov et al. 2013 Nucl. Fusion 53093018

[48] M.W. Chen et al. Fusion Engineering and Design 150 (2020) 111415

[49] N. Fedorczak et al. PFMC 2019 "Infra-red thermography estimation of deposited heat load dynamics on the lower tungsten divertor of WEST"

[50] A. Herrmann et ASDEX U Team. ECA, 25A:2109\{2112\}, 2001

[51] L. Zhang, et al. Nuclear Inst. and Methods in Physics Research, A916 (2019) 169 